\title{
The Impact of Interference from the Side Lanes on mmWave/THz Band V2V Communication Systems with Directional Antennas
}

\author{
Vitaly Petrov, Student Member, IEEE, Joonas Kokkoniemi, Member, IEEE, Dmitri Moltchanov, \\ Janne Lehtomäki, Member, IEEE, Markku Juntti, Senior Member, IEEE, \\ Yevgeni Koucheryavy, Senior Member, IEEE
}

\begin{abstract}
Communications systems operating in the millimeter-wave (mmWave) and terahertz band have been recently suggested to enable high data-rate vehicle-tovehicle communications in $5 \mathrm{G}$ and beyond wireless networks. However, massive deployment of such systems may lead to significant interference, affecting the performance of information transmission. While the multipath interference caused by the signal reflections from the road has been extensively discussed in literature, the interference caused by the vehicles on the side lanes has been insufficiently studied so far. In this paper, using a combination of measurement, simulation, and analytical methods we comprehensively characterize the interference from the side lanes in two typical deployments including highway and urban road environments for millimeter-wave and low terahertz bands. Both the multipath interference and direct interference from the transmitting vehicles on the side lanes is taken into account. As a result of our study, we reveal that: i) the interference from the side lanes can be well approximated using two-dimensional stochastic models without any significant loss of accuracy; and ii) even when highly directional antennas are used there are special spatial configurations, where the interference may greatly affect the performance of the communication systems. We lately apply the developed models to estimate the signal-to-interference ratio and link capacity of mmWave/THz band V2V communications.
\end{abstract}

Index Terms-Vehicle-to-Vehicle communications, Directional antennas, Millimeter waves, THz band, Interference, SIR

\section{INTRODUCTION}

The massive vehicle-to-vehicle $(\mathrm{V} 2 \mathrm{~V})$ communications are envisioned as one of the primary use cases in 5G-graded networks [1]-[3], enabling urgent safety messages exchange [4], collective sensing with high-resolution radars and cameras by (semi-)autonomous vehicles [5]-[8], connectivity to in-vehicle infotainment systems [9], [10], as well as data offloading from the user devices [11]. While the emergency messages exchange over the microwave band is to be implemented already in the next generations of vehicles [12], the latter applications require substantially higher data rates than existing microwave solutions can provide in realistic deployments [13]-[16].

Motivated by the abovementioned applications, the community nowadays develops the V2V communications solutions,

Copyright (c) 2018 IEEE. Personal use of this material is permitted. However, permission to use this material for any other purposes must be obtained from the IEEE by sending a request to pubs-permissions@ieee.org.

This work is supported by Academy of Finland, Infotech Oulu Doctoral Program, and Nokia Foundation.

Vitaly Petrov, Dmitri Moltchanov, and Yevgeni Koucheryavy are with Tampere University of Technology, Tampere, Finland. Email: \{vitaly.petrov, dmitri.moltchanov\}@tut.fi, evgeni.koucheriavy@tut.fi.

Joonas Kokkoniemi, Janne Lehtomäki, and Markku Juntti are with University of Oulu, Oulu, Finland. Email: \{joonas.kokkoniemi, jannel\}@ee.oulu.fi, markku.juntti@oulu.fi. operating over the millimeter waves band (mmWaves, such as $60 \mathrm{GHz}, 77 \mathrm{GHz}$, and $79 \mathrm{GHz}$ [17]-[20]) and also starts to explore the low terahertz $(\mathrm{THz})$ band, in particular, 275$325 \mathrm{GHz}$ [21], as a resource for the future systems. The abovementioned frequencies are characterized by GHz-wide spectrum and, thus, higher capacity [22], [23]. At the same time, they simultaneously call for the need to use directional antenna systems to compensate the greater propagation losses and maintain the desired communication range [24]-[27].

Following the evolution of communications systems, the future vehicles will feature communications modules operating in mmWave/THz frequency bands allowing to exchange massive amounts of data between each other and with the network infrastructure. However, the ubiquitous use of such systems may lead to a significant level of interference. While for the offloaded user traffic the net result is just the lower quality of experience, the loss of the emergency or cruise control messages might put human lives in danger [28], [29].

There have been many studies, analyzing the interference in $\mathrm{V} 2 \mathrm{~V}$ communications systems with directional antennas. For instance, the inter-carrier interference in $\mathrm{V} 2 \mathrm{~V}$ communications has been characterized in [30], [31], however, neither the interference from other vehicles nor the mmWaves $/ \mathrm{THz}$ specifics have been taken into account. The pure measurement-based study of interference in visible light $\mathrm{V} 2 \mathrm{~V}$ communications using headlights was conducted in [32]. The model of interference proposed in [33] focuses exclusively on IEEE 802.11p protocol, operating at $5.9 \mathrm{GHz}$, and, therefore, is not applicable either. There are also several specific models proposed to characterize the multipath interference caused by the signal ground reflections in mmWave $\mathrm{V} 2 \mathrm{~V}$ communication systems, including [34], [35] and many more, however, the interference caused by the vehicles on the side lanes is not taken into account. Summarizing, to the best of the authors' knowledge, the analytical model for the interference caused by the vehicle on the side lanes in V2V communications that simultaneously takes into account (i) the directivity of antennas, (ii) mmWave and $\mathrm{THz}$ waves propagations phenomena, and (iii) the specifics of vehicular deployments have not been proposed yet.

In this paper, we perform the link-layer analysis analysis of mmWave and $\mathrm{THz}$ communications systems with directional antennas operating in $\mathrm{V} 2 \mathrm{~V}$ environment focusing on the interference, signal-to-interference ratio (SIR), and link capacity as the major metrics of interest. To achieve this goal, first, an extensive measurement and simulation campaigns are carried out to investigate the mmWaves/THz waves propagation in typical V2V scenarios. Starting with the measurements of 
$79 \mathrm{GHz}$ and $300 \mathrm{GHz}$ reflection and scattering losses from typical vehicle materials, the study continues with ray-tracing of $\mathrm{V} 2 \mathrm{~V}$ communications with directional antennas. This hybrid measurement/simulation framework allows us to characterize the $\mathrm{mmWave} / \mathrm{THz}$ waves propagation in the vehicular scenario and prioritize the critical effects to be taken into account for the interference modeling. Particularly, we reveal that interference caused by the vehicles on the side lanes can be well approximated by a $2 \mathrm{D}$ stochastic process, while the majority of 3D components can be ignored without the loss of accuracy. We then use these results to develop analytical models for interference in highway and urban deployments and finally present with the link layer performance analysis.

The main contributions of the work are:

- Analysis of interference from the side lanes in mmWave/THz V2V communications with directional antennas: Focusing on the most important propagation effects, two analytical models for the interference in mmWave/ THz waves V2V communications with directional antennas are developed. Both models incorporate the direct interference from the side lane vehicles as well as the interference caused by the multipath due to the signal reflections from the vehicle bodies. The first model is designed for the typical highway scenario, where the distances between vehicles rarely change. The second one is applicable to the urban case, where the distances between the vehicles are random. To the best of the authors' knowledge, this is the first study presenting the measurements-grounded analytical models for interference from the neighbouring vehicles in $\mathrm{mmWaves} / \mathrm{THz}$ $\mathrm{V} 2 \mathrm{~V}$ systems with directional antennas.

- Capacity analysis of mmWave/THz V2V communications in the presence of interference: The developed analytical models are further used in the link layer performance evaluation of $\mathrm{V} 2 \mathrm{~V}$ communications in mmWaves and $\mathrm{THz}$ bands. In particular, the link capacity for both $79 \mathrm{GHz}$ and $300 \mathrm{GHz}$ systems is estimated, taking the interference, noise, and the directivity of antennas into account. The comparison between the two bands is performed. Advantages and shortcomings of $79 \mathrm{GHz}$ and $300 \mathrm{GHz}$ bands with respect to the interference and noise effects on the link capacity are illustrated.

The rest of the paper is organized as follows. Section II introduces the V2V scenarios, path loss, and antenna models. The details of the performed measurements and ray-based simulation campaigns are given in Section III. The analytical models for interference are developed in Section IV, while the numerical results are discussed in Section V. Conclusions are drawn in the last section.

\section{System Model}

In this section, we first introduce the $\mathrm{V} 2 \mathrm{~V}$ scenarios of interest including highway and urban ones, capturing their specifics. Then, we cover the path loss and antenna models used in the further analysis. Finally, we provide the overview of the proposed methodology.

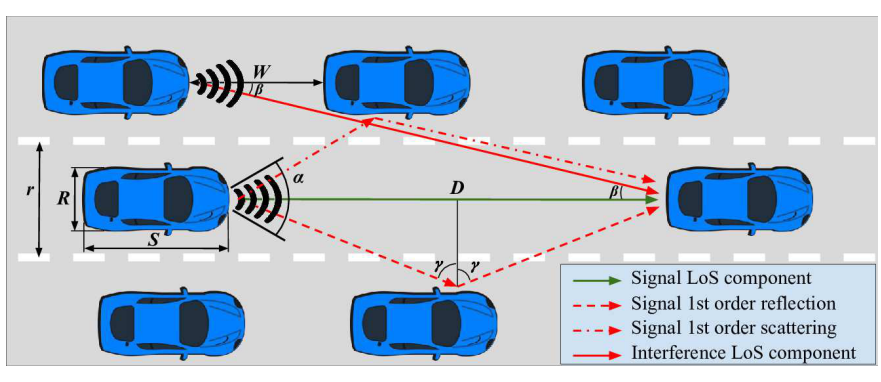

Fig. 1. Top view of the considered deployment.

\section{A. Scenarios and Deployment}

We consider a straight segment of road with three lanes, each with width $r=3.2 \mathrm{~m}$. The considered three-lane segment is occupied with typical size vehicles of dimensions $S \times R \times H$ $(4 \mathrm{~m} \times 1.6 \mathrm{~m} \times 1.8 \mathrm{~m})$ for the length, width, and height, respectively. All the vehicles are assumed to be positioned in the middle of their lanes and equipped with directional antenna systems on the front and rear, so they can establish direct front-to-rear and rear-to-front communications with the neighbouring vehicles [14]. We consider two deployments:

- Deterministic (highway) scenario. In this scenario, the distance between the Tx and Rx vehicles on the central lane is constant and equals to the inter-vehicle distance, $D$. The distance between vehicles on the side lanes, $W$, is also constant. The ratio between $W$ and $D$ can be of any value, thus, modeling different road conditions. This scenario is typical for rural areas and highways [36].

- Stochastic (urban) scenario. In this scenario, we concentrate on communications between two arbitrarily chosen neighboring vehicles moving along the central lane. The distance between any two vehicles on any lane is random. The distributions of $D$ and $W$ are the same and follow an exponential distribution with parameter $\lambda$. This scenario is typical for traffic jams and urban areas [37]. For the sake of simplicity, the buildings and any other obstacles that may potentially appear in the scenario are not modeled.

In both scenarios, the saturated traffic from all the vehicles is assumed, providing the upper bound for the interference level. Although we concentrate on the worst-case interference conditions by considering the link performance on the central lane, the presented analysis is applicable to the vehicles on the side lanes as well. The deployment is shown in Fig. 1.

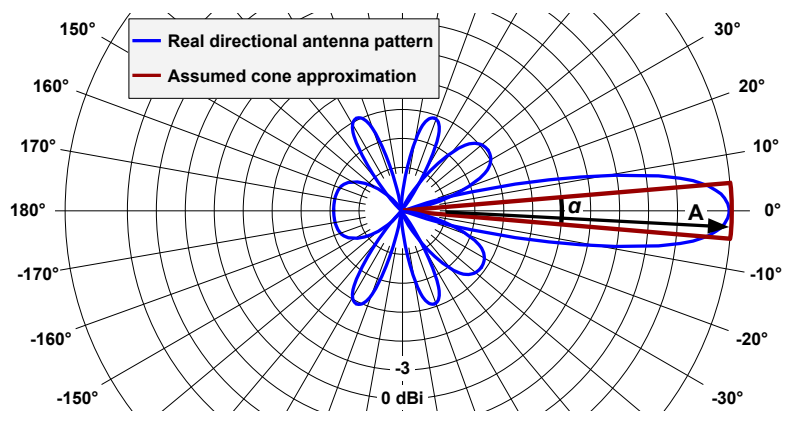

Fig. 2. The considered "cone" antenna model. 


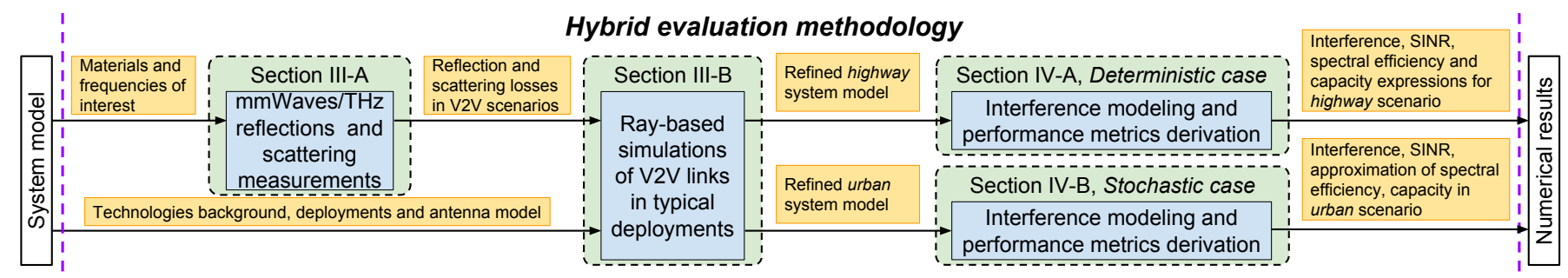

Fig. 3. An overview of the link-layer performance evaluation methodology.

\section{B. Path Loss at Millimeter/Terahertz Frequencies}

The distinguishing feature of $\mathrm{mmWaves} / \mathrm{THz}$ propagation is the presence of the molecular absorption loss [38]-[41]. The loss is caused by molecules having unique absorption spectrum making the wireless channel frequency selective. The link budget for the received power spectral density (psd) is

$$
S_{R x}(f, d)=\frac{S_{T x}(f)}{L_{A}(f, d) L_{P}(f, d)},
$$

where $f$ is the operating frequency, $d$ is the distance between the Tx and the Rx, $S_{T x}(f)$ stands for the transmitted signal psd, $L_{A}(f, d)$ represents the absorption loss, and $L_{P}(f, d)$ is the propagation loss. The absorption loss is defined as [42]

$$
L_{A}(f, d)=\frac{1}{\tau(f, d)},
$$

where $\tau(f, d)$ is the transmittance of the medium following the Beer-Lambert law, $\tau(f, d) \approx e^{-K(f) d}$, where $K(f)$ is the overall absorption coefficient of the medium available from HITRAN database [43]. Thus, the received signal psd is

$$
S_{R x}(f, d)=A d^{-2} e^{-K(f) d},
$$

where $A$ is the coefficient depending on $S_{T x}(f)$ and the antenna model. We derive this coefficient in the following subsection.

\section{Antenna Model}

Following [44], we adopt the "cone" model as an approximation of the antenna radiation pattern. In this model, the directivity of the transmitter is taken into account considering the coverage zone to be cone-shaped, as shown in Fig. 2. This model is an abstraction assuming no side lobes and constant power at a certain separation distance from the transmitter. To parameterize it, we need to provide the coefficient $A$ [45].

The surface area of a wavefront for cone model is given by

$$
S_{A}=\frac{S_{T x}(f)}{S_{R x}(f, d)}=2 \pi d h
$$

where $S_{T x}$ refers to the Tx power spectral density, $S_{R x}(f, d)$ refers to the power density at the wavefront, $h=d[1-$ $\cos (\alpha / 2)]$ and $\alpha$ is the antenna directivity angle. According to free-space propagation loss model, the psd at the wavefront is

$$
S_{R x}(f, d)=\frac{S_{T x}(f)}{S_{A}}=\frac{A d^{-2}}{4 \pi},
$$

leading to the following expression for $A$

$$
A=\frac{2 S_{T x}(f)}{1-\cos (\alpha / 2)} .
$$

\section{Methodology Overview}

The mmWave/THz waves propagation in realistic environments is characterized by a number of phenomena including reflection and diffuse scattering from various objects in the channel. To avoid overcomplicating the analytical models, we initially investigate which particular phenomenon notably affect the link-level performance in the described V2V scenarios. To achieve this goal, in Section III-A we first perform a measurement campaign, report and analyze the characteristics of $\mathrm{mmWave} / \mathrm{THz}$ wave reflection and scattering from the materials inherent for vehicle bodies including aluminium, glass, and hard plastic.

Using the measured data, in Section III-B, we simulate the realistic V2V communications scenario with our custom raytracing simulation tool evaluating the contribution of different signal components to the total amount of power, both useful (signal) and harmful (interference), received by the Rx vehicle. Based on both the measurements and simulation results, we identify the critical effects of the mmWave/ $\mathrm{THz}$ waves propagation in $\mathrm{V} 2 \mathrm{~V}$ communications scenario to be further incorporated in our analytical models.

The interference analysis in the deterministic (highway) scenario, as well as derivation of the associated link-level performance metrics including SINR, spectral efficiency, and capacity, is presented in Section IV-A. The similar study focused on the stochastic (urban) scenario is given in Section IV-B. The methodology is summarized in Fig. 3.

\section{REFLECTIONS AND SCATtERING FROM THE Vehicles}

In this section, we parameterize the system model for $\mathrm{V} 2 \mathrm{~V}$ communications using mmWave and $\mathrm{THz}$ band. First, we use the real measurements campaign to derive reflection/scattering paths gains off the the conventional vehicle materials. Then, using the ray-tracing simulations, we isolate the critical multipath components affecting interference level at the target receiver. These components will be later used in the developed analytical models.

\section{A. Measurement Campaign}

The mmWave/THz communications in $\mathrm{V} 2 \mathrm{~V}$ scenarios are characterized by the special effects that may not exist in conventional open space deployments. Particularly, even in absence of interference from other vehicles, the useful signal at the receiver (LoS component) may be affected by the multipath components, reflected and scattered from the vehicles on the neighboring lanes. Thus, the open space propagation model, 


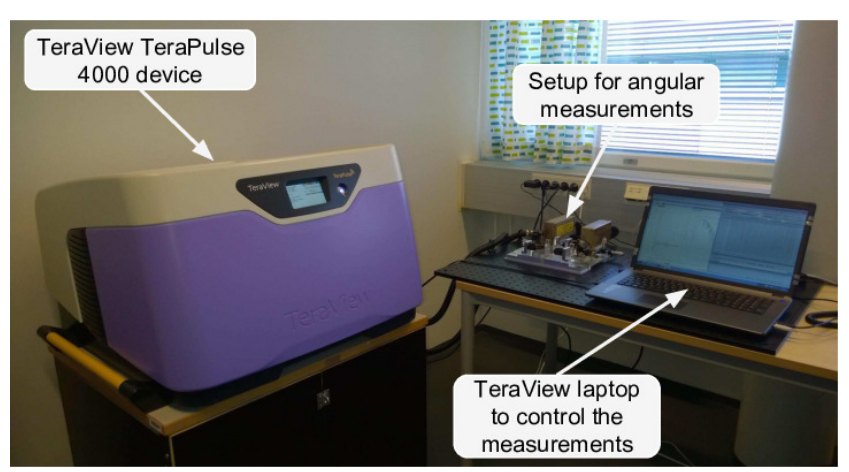

(a) General view

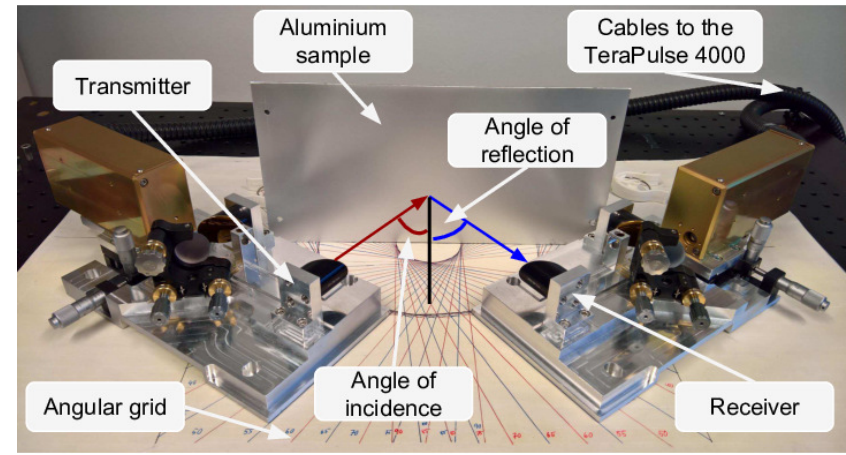

(b) Angular measurements setup

Fig. 4. The THz spectroscopy setup used to measure the reflection and scattering losses.

presented in Section II alone is non-sufficient for interference analysis and needs to be complemented with the reflection and scattering losses from the vehicle bodies.

To characterize the mmWave/ $\mathrm{THz}$ waves propagation in $\mathrm{V} 2 \mathrm{~V}$ scenario, a measurement campaign was conducted. The target parameters were the reflection and scattering losses of the most common materials found on the exterior of modern vehicles: (i) aluminium, typical for wings, hoods, roofs, and door, (ii) tempered glass, typical for windows and headlights and taillights, and (iii) hard plastic, commonly used in the bumpers, door moldings, side skirts, and fenders. The measurements for both $79 \mathrm{GHz}$ and $300 \mathrm{GHz}$ were performed on a single setup consisting of (see Fig. 4): (i) a mmWave/THz band measurement platform, (ii) a single transmitter and a single receiver, placed $20 \mathrm{~cm}$ from each other, (iii) three samples of different materials $3 \mathrm{~mm}$ thin each, (iv) several holders, and (v) a marked-up map for accurate angular measurements.

The measurement campaign has been performed in three stages. First, the signal attenuation was measured for all three materials as a function of the angle of incidence and the $3 \mathrm{D}$ angle of reflection/scattering. Then, the reference signal attenuation for the LoS path of the same distance was measured. Finally, to estimate the relative signal loss, caused exclusively by the reflection and scattering, the signal attenuations measured during the first stage were scaled with the reference signal attenuation, measured at the second stage. This method, by design, gives us the extra signal attenuation as a function of the selected material and three $2 \mathrm{D}$ angles (the angle of incidence and the angle of reflection/scattering in two planes), which is further used in the ray-tracing simulator.

To measure the signal losses, we used the TeraView TeraPulse 4000 [46] platform, based on $\mathrm{THz}$ time domain spectroscopy (THz-TDS). In this device, the full time domain detection window consists of multiple short time duration windows, determining the resolution time and, thus, the accuracy of the frequency-dependent metrics. The signal amplitude in this device is detected in one short window at a time. By repeating this step, while delaying the reception, the full time domain signal is captured. In our measurement, we set the resolution time to $100 \mathrm{fs}$ and composed the received signal from $10^{4}$ samples. Consequently, the total detection window was slightly over $1 \mathrm{~ns}$. To reduce the noise level, the results were averaged over 100 trials for all the combinations of angles. The received time domain picture was then translated to the frequency domain to estimate the signal attenuation at different frequencies. As the total bandwidth of the transmitted pulse in TeraView TeraPulse 4000 is $5 \mathrm{THz}$, the abovementioned set of parameters leads to the frequency resolution of around $1 \mathrm{GHz}$, which gives a sufficiently accurate picture for our study.

Due to the spatial limitations of the setup, the measurements were made only for the angles from $35^{\circ}$ to $75^{\circ}$. This range is sufficient as the smaller angles of an incident to the side lane vehicles are not usually observed when directional antennas are used. Moreover, the substantial signal attenuation at higher angles makes the contribution of such paths negligible.

The examples of the measurement results for $79 \mathrm{GHz}$, illustrating the reflection and scattering attenuations of the glass, plastic, and aluminium, are shown in Fig. 5. Aluminium is, expectedly, the best reflector of the three selected materials with the reflection loss been in the order of $0-2 \mathrm{~dB}$, regardless the angle of incident. At the same time, the attenuation, when the transmitter angle is different from the receiver angle (scattering loss), is substantially higher. On the contrast, the rest two materials are characterized by a significant dependency between the reflected path gain and the angle of incident. The reflection loss for glass increases from $0 \mathrm{~dB}$ to $\approx 10 \mathrm{~dB}$ from $35^{\circ}$ to $75^{\circ}$, while the plastic sample almost stopped reflecting any notable amounts of energy after $50^{\circ}$, while the difference between the reflection loss at $35^{\circ}$ and $75^{\circ}$ raises to $\approx 22 \mathrm{~dB}$.

Analyzing the results for $300 \mathrm{GHz}$, presented in Fig. 6, we notice that this frequency is characterized by larger losses for both reflection and scattered rays, while the qualitative behavior is mostly similar. The aluminium sample is nearly unaffected by the incident angle with approximately $0 \mathrm{~dB}$ path gain at $35^{\circ}$ angle, and decreasing to $-3 \mathrm{~dB}$ at $70^{\circ}$. The same figures for the glass report $-2.5 \mathrm{~dB}$ and $-13.2 \mathrm{~dB}$ path gains for the reflected rays with $35^{\circ}$ and $75^{\circ}$ angles of incident, respectively. The corresponding path gain values for hard plastic are $-7.3 \mathrm{~dB}$ and $-14.6 \mathrm{~dB}$.

\section{B. Ray-Tracing Assessment}

To understand the contribution of LoS, reflection and scattering paths into the received signal strength we now present the results of ray-tracing simulations for different distances 


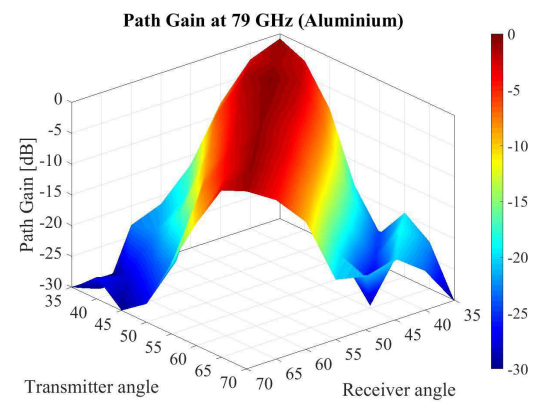

(a) Path gain at $79 \mathrm{GHz}$ for aluminium

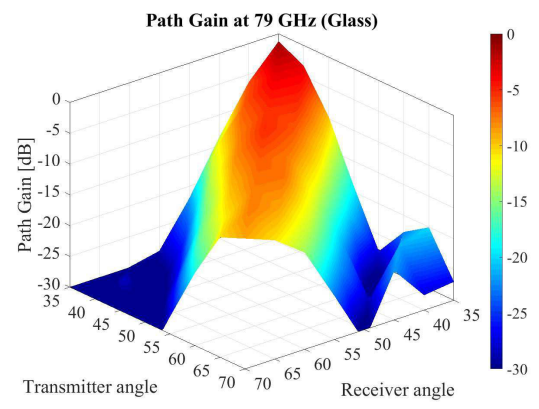

(b) Path gain at $79 \mathrm{GHz}$ for glass

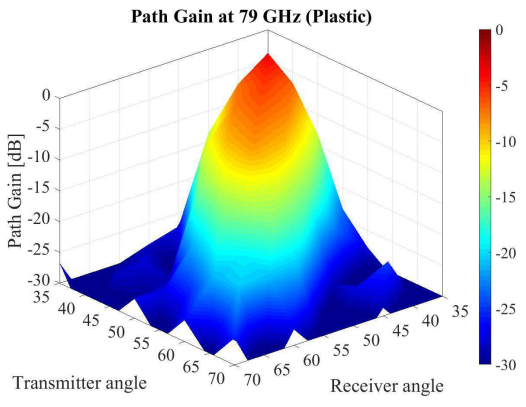

(c) Path gain at $79 \mathrm{GHz}$ for plastic

Fig. 5. Reflection/scattering path gains at $79 \mathrm{GHz}$ for typical vehicle body materials.

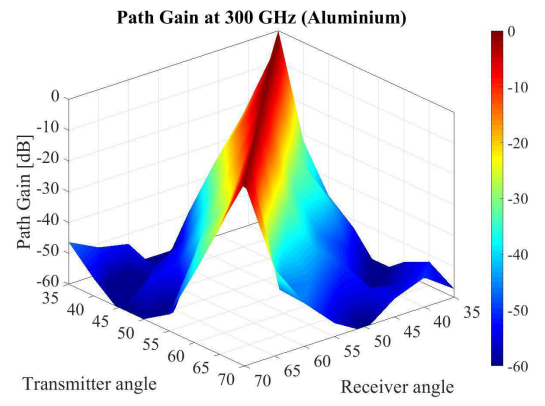

(a) Path gain at $300 \mathrm{GHz}$ for aluminium

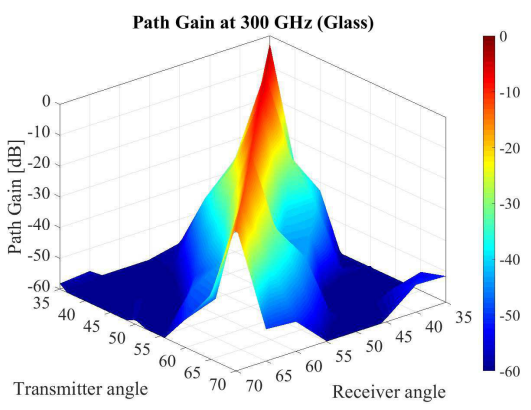

(b) Path gain at $300 \mathrm{GHz}$ for glass

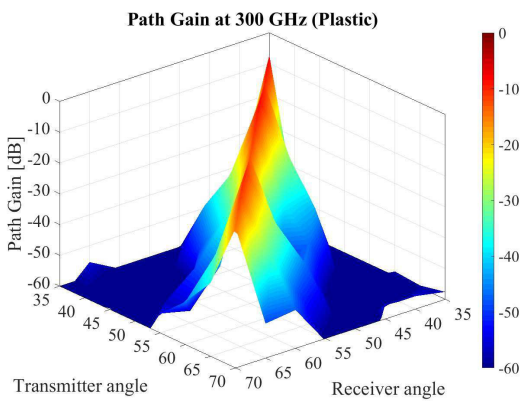

(c) Path gain at $300 \mathrm{GHz}$ for plastic

Fig. 6. Reflection/scattering path gains at $300 \mathrm{GHz}$ for typical vehicle body materials.

between the transmitting and the receiving vehicle, surrounded by other vehicles on the neighboring lanes.

The commercially available ray-tracing simulators do not allow for parameterization of the considered scenarios with the measured attenuation coefficients. Most of them also do not take into account scattering of orders higher than one. For this reason, we have developed our own ray-tracing simulator, supporting both abovementioned features. The simulator is written in $\mathrm{C}++$ and is able to simulate around 1.5 billions of rays per minute, thus, enabling simulation of second- and third-order scattering within a reasonable time. The algorithm is based on the surface tessellation to miniature segments of the size comparable to the wavelength. Each of these segments is considered as either a point receiver for the incoming ray and a point transmitter for the outgoing ray. The simulator captures all the possible ray geometries, attenuates each of the ray energies with both propagation loss and reflections/scattering losses and, finally, aggregates all the received rays to compare

TABLE I

PARAMETERS FOR THE RAY-TRACING SIMULATIONS.

\begin{tabular}{|l|l|}
\hline Parameter & Value \\
\hline \hline Operation frequency & $0.3 \mathrm{THz}$ \\
\hline Tx/Rx antenna gains & $20 \mathrm{~dB}$ \\
\hline Tx/Rx antenna directivity angle & $30^{\circ}$ \\
\hline Antenna altitude & $0.2 \mathrm{~m}$ \\
\hline Vehicle length & $4 \mathrm{~m}$ \\
\hline Vehicle height & $1.6 \mathrm{~m}$ \\
\hline Distance between vehicles on left and right lanes & $1 \mathrm{~m}$ \\
\hline Offset to the left lane & $0.2 \mathrm{~m}$ \\
\hline Offset to the right lane & $0.3 \mathrm{~m}$ \\
\hline
\end{tabular}

their impact in terms of the received power.

In this study, we analyze the "worst-case" scenario, simulating communications in dense $\mathrm{V} 2 \mathrm{~V}$ traffic conditions in saturation. The distance between the vehicles on neighboring lanes is constant and set to $W=1 \mathrm{~m}$, while the distance between the Tx and $\mathrm{Rx}$ vehicles, $D$, is also constant, but ranges from $10 \mathrm{~m}$ to $25 \mathrm{~m}$. The combination of large distance between Tx and Rx vehicles and the low distance between vehicles on the neighboring lanes leads to the substantial amount of possible multipath routes. The scenario is illustrated in Fig. 7, while the simulation parameters are shown in Table I.

Both transmitter and receiver are located on the typical radar positions, i.e., the transmitter is on the vehicle front bumper and receiver is placed on the other car's back bumper. The altitude of both is $0.2 \mathrm{~m}$. The vehicles are assumed to be $4 \mathrm{~m}$ long and $1.6 \mathrm{~m}$ high. To allow for more reflection and scattering rays, the vehicles on the neighboring lanes are at $0.2 \mathrm{~m}$ and $0.3 \mathrm{~m}$ offsets from the transmitting car. Both Tx and $\mathrm{Rx}$ antennas are assumed to be $30^{\circ}$ wide, which results in $\approx 17.8 \mathrm{~dB}$ gains, following the antenna pattern from Section II. The transmit power is assumed to be equal for both bands and set to $20 \mathrm{dBm}$. The vehicle side is approximated with a rectangle that starts at $0.2 \mathrm{~m}$ altitude. It is made of plastic from $0.2 \mathrm{~m}$ to $0.3 \mathrm{~m}$, then, from aluminium between $0.3 \mathrm{~m}$ and $1.1 \mathrm{~m}$, and, finally, from glass for $1.1 \mathrm{~m}$ to $1.6 \mathrm{~m}$. Although in real scenarios the dimensions and shape of vehicles might vary, the considered "average" scenario provide the first-order approximation for $\mathrm{mmWaves} / \mathrm{THz}$ propagation allowing to assess the importance of propagation phenomena.

The obtained received power for different distances between 


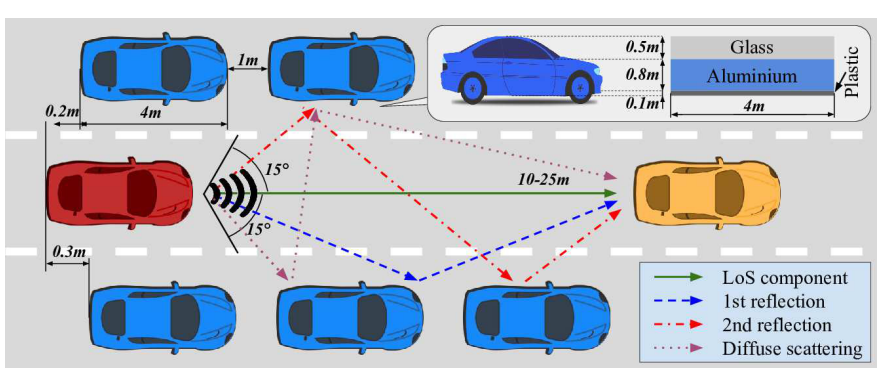

Fig. 7. Ray-tracing simulation scenario.

Tx and Rx are shown in Fig. 8. As one may observe, while the first-order reflection is present for all the distances, the second-order reflection appears only for the largest one. The reason is that for the considered antenna angle of $30^{\circ}$, the minimal distance for the second reflection to appear, $D_{\min }$, is

$$
D_{\text {min }}=4 \frac{r / 2}{\tan (\alpha / 2)}=\frac{2 r}{\tan (\alpha / 2)} \approx 24 \mathrm{~m},
$$

where $r=3.2 \mathrm{~m}$ is the lane width, and $\alpha$ is the antenna angle. For values of $D$, the second-order reflection from the side lane vehicles is not feasible. Similarly, the first-order reflection may appear starting from $D \approx 12 \mathrm{~m}$.

The second observation from Fig. 8 is that, regardless of the distance between Tx and Rx, even in the dense scenario with many scattered rays, the aggregated contribution of scattered rays is significantly smaller than that of the reflected paths.

The presented results allow to make the following conclusions: (i) the LoS component is the strongest for all the considered separation distances between $\mathrm{Tx}$ and $\mathrm{Rx}$, (ii) the diffuse scattering does not provide any noticeable effect on the received energy, (iii) the energy coming from the first-order reflections is notable and has to be taken into account in both urban highway scenarios, (iv) the contribution of second- and higher-orders reflections has to be considered only in highway scenario, when the distance between the Tx and Rx vehicles is large, and (v) the abovementioned conclusions are equally applicable to both $79 \mathrm{GHz}$ and $300 \mathrm{GHz}$ center frequencies.

The performed ray-tracing simulations allowed us to significantly simplify the system models for both urban and highway scenarios, avoiding the need for analytical modeling of the rays, scattered from the side lane vehicles. Thus, only the LoS and the first-order reflection will be considered in the urban scenario analysis, while the higher order reflections will also be taken into account in the highway scenario. Moreover, the substantial difference between the LoS component and the reflected rays motivates us to simplify the analysis even further and consider only the LoS components in the aggregated interference from the side lane vehicles.

\section{INTERFERENCE IN MMWAVES/THZ V2V SYSTEMS}

In this section, we develop the analytical models for both the highway and urban scenarios in mmWave/THz V2V systems. The metrics of interest are the mean interference, SINR, spectral efficiency, and link capacity estimation.

\section{A. Deterministic (Highway) Scenario Analysis}

In this section, we develop the analytical model for interference in highway scenario with constant distances between the vehicles. We first estimate the mean multipath interference, caused by the reflections and then analyze the mean interference from the other side lane vehicles. Finally, we derive approximations for the mean SINR, spectral efficiency, and link capacity.

1) Multipath Interference: Recalling that the scattering field is much weaker compared to reflected paths, multipath interference is mostly caused by $n$ th-order reflections. Observing the structure of the multipath interference in the considered scenario one could notice that the presence of reflections depends on the distance $D$, and antenna angle $\alpha$. There could be at most two reflections of order $n$. Thus, the multipath interference is a piecewise continuous function of $D$. Below, we first determine the distances such that the $n$th order reflections may exist and then estimate their probabilities.

Observing Fig. 9(a) we see that for the first reflection to exist the following needs to be satisfied

$$
D-\frac{2 r}{\tan \alpha}=L>0 \text {. }
$$

where $\alpha$ is the antenna angle.

In general, if the following holds

$$
\frac{2 r n}{\tan \alpha}<D<\frac{2 r(n+1)}{\tan \alpha},
$$

there could be up to $n$ reflections.

The traveled distance of the first-order reflection is

$$
V_{1}=2 V=2 \sqrt{r^{2}+\left(\frac{D}{2}\right)^{2}} .
$$

The $n$ th-order reflection traveled distance is

$$
V_{n}=2 \sqrt{r^{2}+\left(\frac{D}{2 n}\right)^{2}}+\frac{(n-1) D}{n}
$$

Note that (9) is not sufficient for reflections to exist. Indeed, for the $n$th order reflection to exist, there should be vehicles reflecting the signal at certain places. To take this effect into account we observe that the side lane vehicles are not perfectly aligned on their lanes. The fraction of line on left and right lanes "covered" with vehicles is $q=S /(S+W)$. Due to the randomness in the alignment of vehicles, the probability of having a first-order reflection from left and right lanes is: (i) independent due to independent shifts of the left and right lanes with respect to the central lane and (ii) happen with probability $q$. Note that the presence of the second-order reflections depends on the fact whether there exist the first-order reflections. However, taking into account the abovementioned randomness in the positions of vehicles, the probabilities of having an $n$ th-order reflection can be approximated by $q^{n}$. Observe that this probability depends on the antenna directivity angle $\alpha$ and is conditioned on the distance between communicating vehicles obtained in (9). Thus, in spite of the constant distance between Tx and Rx, 


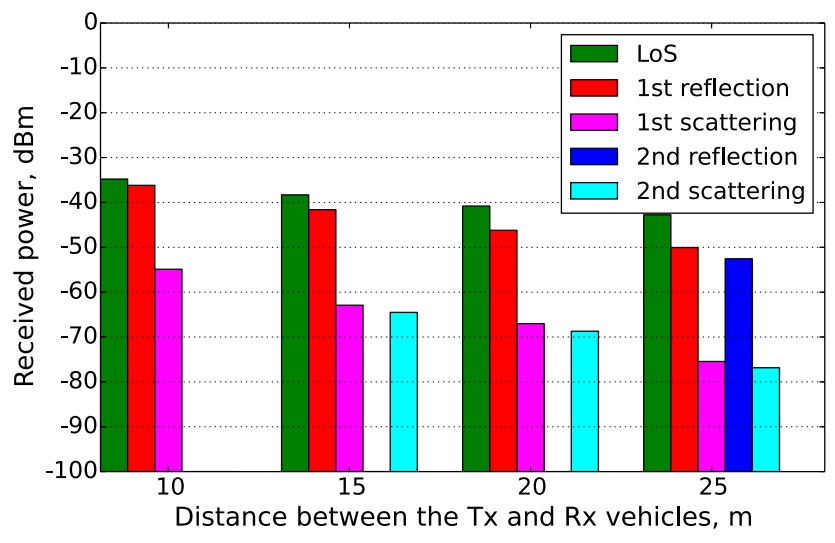

(a) $79 \mathrm{GHz}$ center frequency

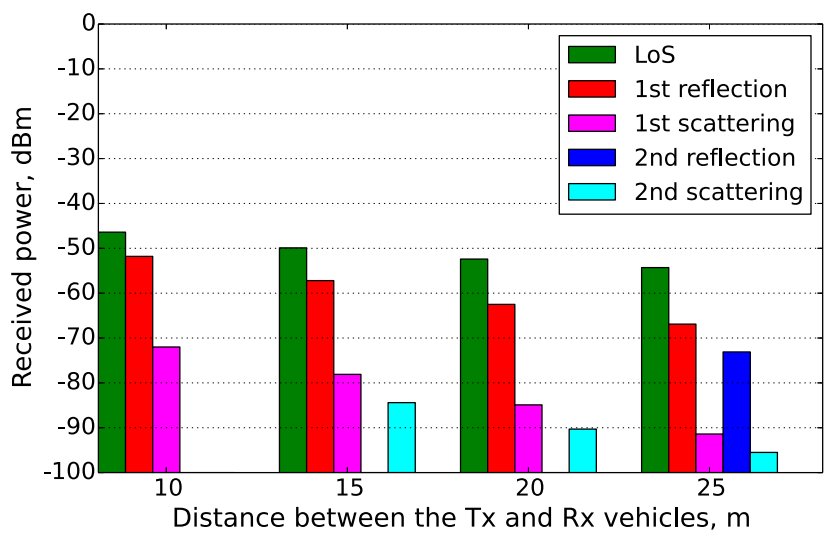

(b) $300 \mathrm{GHz}$ center frequency

Fig. 8. Contribution of the different components to the total received power obtained via ray-based simulations.

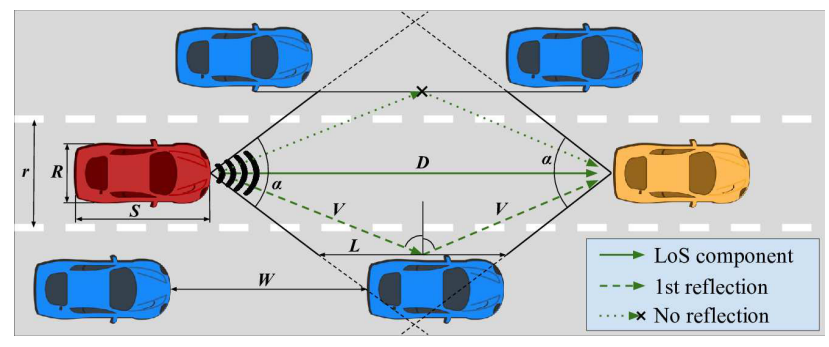

(a) Scenario geometry

Fig. 9. Illustrations of the considered V2V communications scenario.

$D$, the multipath interference is a random variable. Its mean value can be found as

$$
E\left[P_{I}\right]=2 A \sum_{i=1}^{n} q^{i} \frac{V_{i}^{-2} e^{-K V_{i}}}{i k},
$$

where $k$ is a coefficient accounting for attenuation due to reflections. The values of this coefficient depend on the operational frequency, type of the vehicle material, the angle of incidence, and the angle of reflection. The numerical value of this coefficient is obtained via field measurements and is shown in Fig. 5 and Fig. 6.

The presented analysis can be extended to capture various specifics of the considered scenario. First of all, the developed model can be applied to analyze the interference not only at the middle lane (worst-case in our study) but also at the side lane. For the side lane case, (12) reduces to $E\left[P_{I}\right]=A q V_{1}^{-2} e^{-K V_{1}} / \mathrm{k}$, if no buildings or safety barriers on the roadsides are assumed since the second and beyond order reflections do not exist.

In addition, the model can be extended to account for difference densities of cars on the neighbouring lanes. Here, one needs to account for different probabilities of reflections both neighbouring lanes, $q_{L}$ and $q_{R}$. Thus, (12) extends to

$$
\begin{aligned}
E\left[P_{I}\right] & =A\left(q_{L} \frac{V_{1}^{-2} e^{-K V_{1}}}{k}+\sum_{i=1}^{n} q_{L}^{\left\lceil\frac{i}{2}\right\rceil} q_{R}^{\left\lfloor\frac{i}{2}\right\rfloor} \frac{V_{i}^{-2} e^{-K V_{i}}}{i k}\right)+ \\
& +A\left(q_{R} \frac{V_{1}^{-2} e^{-K V_{1}}}{k}+\sum_{i=1}^{n} q_{R}^{\left\lceil\frac{i}{2}\right\rceil} q_{L}^{\left\lfloor\frac{i}{2}\right\rfloor} \frac{V_{i}^{-2} e^{-K V_{i}}}{i k}\right) .
\end{aligned}
$$

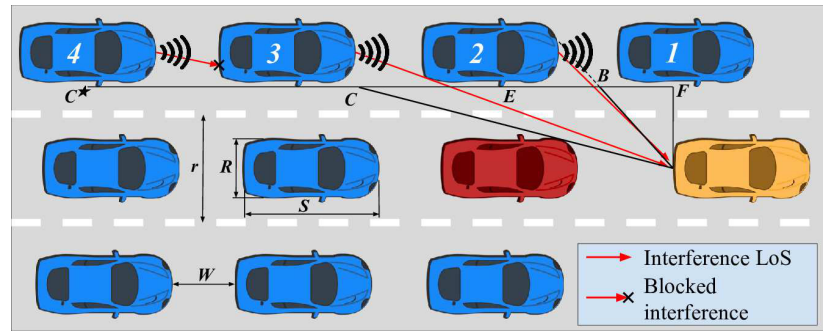

(b) Interference

2) Interference from Other Vehicles: The vehicles on the side lanes do induce interference to the Rx via LoS paths if and only if the distance between them, $W$, is less than $R / 2 \tan \alpha$. Otherwise, the signal is reflected/scattered in reverse direction. From now on we concentrate on the case $W>R / 2 \tan \alpha$ and estimate the interference induced by the LoS paths only as their contribution is much stronger compared to reflected paths. The interference caused by reflections can be calculated similarly to the previously presented multipath interference analysis. We also neglect the interference caused by reflected paths from vehicles behind Tx as its contribution is expected to be low due to substantial pathloss.

Consider Fig. 9(b) and define $C B$ as the interference interval for the Rx on the left lane. This segment contains the vehicles producing interference via direct LoS path between them and Rx. In Fig. 9(b) these vehicles are labeled as 2 and 3. This interference interval is the minimum of two segments $C^{\star} B$ and $C B$, where the starting point of the former is dictated by the distance between vehicles on the side lanes, $W$, while the latter is defined by the distance between Tx and $\mathrm{Rx}, D$.

Consider first $C^{\star} B$. The farthest point $C^{\star}$, where vehicles on the side lane may produce interference via $\operatorname{LoS}$ paths is defined by the angle $\gamma=\tan ^{-1}(R / 2 W)$. Observe that $\left\|C^{\star} B\right\|=$ $\left\|C^{\star} D\right\|-\|B F\|$. The latter component is readily given by $\|B F\|=r \tan (\pi / 2-\alpha)$, while the distance $\|C F\|$ is

$$
\left\|C^{\star} F\right\|=r \tan \left[\frac{\pi}{2}-\tan ^{-1}\left(\frac{R}{2 W}\right)\right],
$$

where $R$ is the width of a car. 
Combining the latter results we get

$$
\left\|C^{\star} B\right\|=r\left[\tan \left(\frac{\pi}{2}-\tan ^{-1}\left(\frac{R}{2 W}\right)\right)-\tan \left(\frac{\pi}{2}-\alpha\right)\right],
$$

and simplifying, we arrive at

$$
\left\|C^{\star} B\right\|=r\left(\frac{2 W}{R}-\cot \alpha\right) .
$$

The distance $\|C B\|$ is obtained similarly, i.e.,

$$
\|C B\|=r\left(\frac{2 D}{R}-\cot \alpha\right),
$$

providing the interference interval as $\min \left(\left\|C^{\star} B\right\|,\|C B\|\right)$.

To address the worst case interference case we assume that the first interfering vehicle is located at point $B$. The number of vehicles falling into the interference interval is given by

$$
N=\left\lfloor\frac{\min \left(\left\|C^{\star} B\right\|,\|C B\|\right)}{S+W}\right\rfloor,
$$

where $\lfloor\cdot\rfloor$ is the floor function, $S$ is the length of a car.

The interference path lengths are now given by

$$
V_{i}^{\star}=\sqrt{\left[r \tan \left(\frac{\pi}{2}-\alpha\right)+i(S+W)\right]^{2}+r^{2}} .
$$

The interference caused by other vehicles is

$$
E[I]=2 A \sum_{i=1}^{N}\left(V_{i}^{\star}\right)^{-2} e^{-K V_{i}^{\star}}
$$

3) Link-Level Performance Metrics: The mean of the total interference can be obtained as the sum of multipath interference provided in (12) and interference created by other vehicles obtained in (20), that is,

$$
\begin{aligned}
E[I] & =E\left[P_{I}\right]+E[I]= \\
& =2 A\left(\sum_{i=1}^{n} q^{i} \frac{V_{i}^{-2} e^{-K V_{i}}}{i k}+\sum_{i=1}^{N}\left(V_{i}^{\star}\right)^{-2} e^{-K V_{i}^{\star}}\right) .
\end{aligned}
$$

The mean value of SINR is then

$$
E[S]=\frac{e^{-K D} D^{-2}}{N(B)+2\left(\sum_{i=1}^{n} q^{i} \frac{V_{i}^{-2} e^{-K V_{i}}}{i k}+\sum_{i=1}^{N}\left(V_{i}^{\star}\right)^{-2} e^{-K V_{i}^{\star}}\right)},
$$

where $N(B)$ is the total thermal noise.

Consider the spectral efficiency, $U$, and link capacity, $C$,

$$
U=f(x)=\log _{2}(1+S), C=g(x)=B \log _{2}(1+S) .
$$

To obtain moments of $U$ and $C$ we need the distribution of SINR. However, using the Taylor expansion approximation of $g(x)$ and $f(x)$ we can show that

$$
\begin{aligned}
& E[U]=\sum_{i=0}^{\infty} \frac{f^{(i)}(E[S])}{i !} E\left[(S-E[S])^{2}\right], \\
& E[C]=\sum_{i=0}^{\infty} \frac{g^{(i)}(E[S])}{i !} E\left[(S-E[S])^{2}\right],
\end{aligned}
$$

where $g^{(i)}$ and $f^{(i)}$ denote $i$ th derivatives of $f(x)$ and $g(x)$, respectively. Thus, the first-order approximations for mean spectral efficiency and link capacity can be obtained as

$$
E[U] \approx \log _{2}(1+E[S]), E[C] \approx \sum_{f=B_{\min }}^{B_{\max }} \log _{2}(1+E[S(f)]),
$$

where $B_{\min }$ and $B_{\max }$ are the minimum and maximum frequencies of the channel such that $B=B_{\max }-B_{\min }, E[S(f)]$ is the mean SINR value corresponding to frequency $f$. Deriving the second moment of interference one can improve the accuracy of approximation by including the second moment in (24).

\section{B. Stochastic (Urban) Scenario Analysis}

Consider now the stochastic (urban) scenario. Recall that according to our assumptions the distance between any two vehicles on any lane, $W$, is a random variable. The interference analysis for this case is much more complicated. Indeed, the distances $V_{i}, i=0,1, \ldots, n$ affecting the contributions of LoS and $n$th reflected paths components are now random variables. In addition, the number of vehicles providing interference as well as distances $V_{i}^{\star}, i=0,1, \ldots$, are all random variables as well and they depends on the random distance between communicating vehicles. Thus, to address the stochastic case we need to obtain the joint distribution function of $M+2 N+1$ components, where $M$ is the number of reflections, $N$ is the number of vehicles contributing to the interference. Thus, in what follows, we reply upon the series of approximations to provide estimates of the mean performance metrics.

1) Useful Received Signal Strength and Multipath Interference: Following our assumptions, the probability density function (pdf) of the distance between communicating vehicles is exponentially distributed with parameter $\lambda=1 / E[W]$. To obtain the LoS contribution we use the random variables (RV) transformation technique [47]. Observing that $A D^{-2} e^{-K D}$ is the function of a single RV, the density of URSS is written as

$$
f_{P}(y)=\sum_{\forall i} f(\psi(y))\left|\psi^{\prime}(y)\right|,
$$

where $x=\psi(y)=\phi^{-1}(x)$ is the inverse function.

The inverse of $y=\phi(x)=A e^{-K x} x^{-2}$ has two branches

$$
x_{1, i}=\psi(y)=\frac{2 \Theta\left( \pm \frac{K}{2} \sqrt{\frac{A}{y}}\right)}{K},
$$

where $\Theta(\cdot)$ is the Lambert function [48]. The modulo of derivative of the branch of interest is

$$
\left|\psi^{\prime}(y)\right|=\left|\frac{1}{K y\left[\Theta\left(-\frac{K}{2} \sqrt{\frac{A}{y}}\right)+1\right]}-\frac{1}{K y}\right| .
$$

Substituting (28) and (27) into (26) we get URSS pdf as

$$
f_{P}(y)=\lambda e^{-\lambda \frac{2 \Theta\left(-\frac{K}{2} \sqrt{\frac{A}{y}}\right)}{K}}\left|\frac{1}{K y\left[\Theta\left(-\frac{K}{2} \sqrt{\frac{A}{y}}\right)+1\right]}-\frac{1}{K y}\right|,
$$

that can now be used to estimate the mean URSS, $E[P]$. 
2) Multipath interference: Consider now the multipath interference, caused by the first-order reflection, $P_{I}$. The first reflected path may exist only if $W \geq 2 r / \tan \alpha$. Consider the pdf of multipath interference conditioned on the event $\{A: W \geq 2 r / \tan \alpha\}$. To obtain the joint distribution of the distances $D$ and $D_{1}$ observe that $D_{1}$ is related to $D$ as

$$
D_{1}=\sqrt{D^{2}+4 r^{2}} .
$$

The inverse function of (30) is $x_{1, i}=\psi(y)= \pm \sqrt{r^{2}-4 y^{2}}$. The derivative of the first branch is $\psi^{\prime}(y)=-4 y / \sqrt{r^{2}-4 y^{2}}$. Recalling exponential assumption the pdf of $D_{1}$ is

$$
f_{D_{1}}(y)=\frac{4 \lambda y}{\sqrt{r^{2}-4 y^{2}}} e^{-\lambda \sqrt{r^{2}-4 y_{1}^{2}}}
$$

The joint pdf of $D_{1}$ and $D$ is then

$$
f_{D, D_{1}}\left(y_{0}, y_{1}\right)=\frac{4 \lambda^{2} y_{1}}{\sqrt{r^{2}-4 y_{1}^{2}}} e^{-\lambda\left(y_{0}+\sqrt{r^{2}-4 y_{1}^{2}}\right.} .
$$

Once the joint pdf is found we determine the Jacobian of the transformation $f\left(y_{0}, y_{1}\right)=A\left[D^{-2} e^{-K D}+D_{1}^{-2} e^{-K D_{1}}\right]$. Then, the joint pdf of $P$ and $P_{1}$ is obtained according to [47]. The partial derivatives for this transformation are given by

$$
\begin{aligned}
& \frac{\partial f\left(y_{0}, y_{1}\right)}{\partial y_{0}}=A\left(-\frac{2 e^{-K x}}{x^{3}}-\frac{K e^{-K x}}{x^{2}}\right), \\
& \frac{\partial f\left(y_{0}, y_{1}\right)}{\partial y_{1}}=A\left(-\frac{2 e^{-K y}}{y^{3}}-\frac{K e^{-K y}}{y^{2}}\right),
\end{aligned}
$$

and, thus, the Jacobian takes the following form

$$
J=\left|\begin{array}{cc}
1 & 1 \\
A\left(-\frac{2 e^{-K x}}{x^{3}}-\frac{K e^{-K x}}{x^{2}}\right) & A\left(-\frac{2 e^{-K y}}{y^{3}}-\frac{K e^{-K y}}{y^{2}}\right)
\end{array}\right|
$$

The mean multipath interference can now be obtained as

$$
E\left[P_{I}\right]=\int_{0}^{\infty} y\left(\int_{0}^{\infty} f_{P, P_{I}}(x, y) d x\right) d y
$$

where $f_{P, P_{1}}(x, y)$ is the joint pdf of $P$ and $P_{I}$.

3) Interference from Other Vehicles: We now approximate the interference produced by other vehicles by taking into account the interference caused by the LoS paths neglecting the reflected components. Recall that the interference interval in deterministic scenario is $\min \left(\left\|C^{\star} B\right\|,\|C B\|\right)$, where the coordinate of $C^{\star}$ and $C$ are determined by the blocking of LoS by the vehicle of the left or right lanes of the or by the Tx. In stochastic case, the interference interval is fully determined by the distance between Tx and Rx [49], [50]. When $D$ follows exponential distribution, according to [47], the length $\|C B\|$ is obtained as

$$
\psi(y)=\frac{R(y+r \cot \alpha)}{2 r},\left|\psi^{\prime}(y)\right|=\frac{R}{2 r},
$$

resulting in the following density

$$
f_{\|C B\|}(y)=\frac{\lambda R}{2 r} \exp \left(-\lambda \frac{R(y+r \cot \alpha)}{2 r}\right) .
$$

Once the distribution of the interference interval is obtained, we proceed with determining the number of vehicles producing the interference. Recall that the number of points in any closed interval of length $L$ of the Poisson point process with intensity $\lambda$ follows Poisson distribution with parameter $\lambda\|C B\|$. Although the intervals between vehicles are assumed to be exponentially distributed, the process of vehicles is not Poisson due to their non-infinitesimal lengths, $S$. To obtain the number of vehicles producing interference we thin the original Poisson process of centers of vehicles using the coefficient $p$

$$
p=\frac{1 / \lambda}{1 / \lambda+S}=\frac{1}{1+s \lambda}
$$

and assume that the resulting process of vehicles is Poisson with intensity $p \lambda$. Thus, the number of vehicles falling into interference interval of length $\|C B\|$ is Poisson with intensity $p \lambda L$. Since $\|C B\|$ is random with pdf provided in (37) we have the following for the probability of having $k$ vehicles in an interference interval

$$
\operatorname{Pr}\{N=k\}=\int_{0}^{\infty} \frac{(p \lambda x)^{k}}{k !} e^{-p \lambda x} f_{\|C B\|}(x) d x,
$$

that can be computed numerically. Since vehicles on the left and right lanes are assumed to be positioned independently this result holds for both lanes.

Finally, we have to determine the interference distances $V_{i}^{\star}, i=1,2, \ldots$. Since there is no straightforward way to determine their distributions we assume that the all $V_{i}^{\star}$ shares the common interference distance $\|A E\|$, where the point $E$ is the center of the segment $\|C B\|$. The rationale behind this approach is that choosing this approximations the contributions of vehicles with different distances evens out. The mean value of $\|C B\|$ is obtained numerically from (37) and the common interference distance is then

$$
\|A E\|=\sqrt{(\|E F\|+\|B F\|)^{2}+r^{2}} .
$$

Now the interference created by LoS paths is written as

$$
I=A\left(\sum_{i=1}^{N} e^{-K\|\mid A E\|}(|| A E||)^{2}+\sum_{i=1}^{N} e^{-K\|A E\|}(\|A E\|)^{2}\right),
$$

where we have two components in the brackets to take into account the effect of both lanes. Recalling that $\|A E\|$ is the function of the mean of a random variable $\|C B\|$ and, thus, constant, the only random variable is $N$.

Rearranging the terms in (41) as

$$
\begin{aligned}
I & =A \sum_{i=1}^{N+N} e^{-K\|A E\|}(\|A E\|)^{-2}= \\
& =A(N+N) e^{-K\|A E\|}(\|A E\|)^{-2},
\end{aligned}
$$

where $N+N$ is the sum of two discrete random variables representing the number of interfering vehicles whose mass function has been obtained in (39).

Taking expectations from the both sides of (42) the mean interference created by LoS components is readily given by

$$
E[I]=2 A E[N] e^{-K\|A E\|}(\|A E\|)^{-2} .
$$




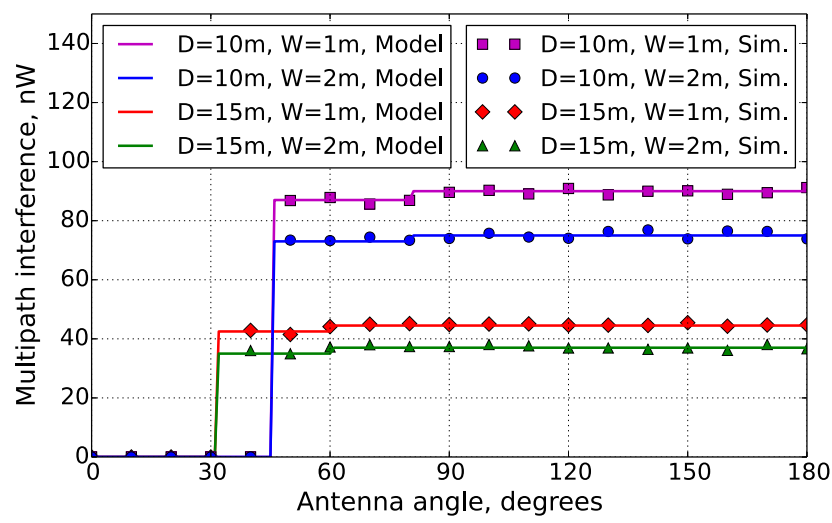

(a) Mean multipath interference

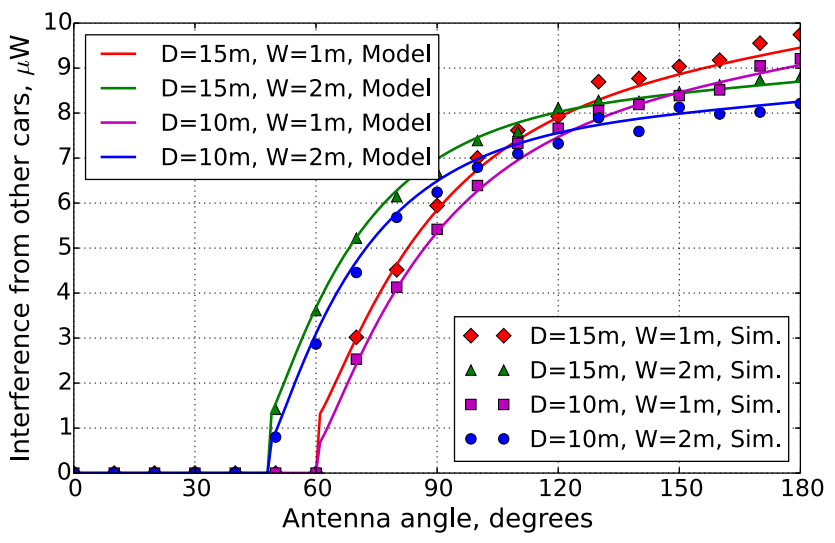

(b) Mean interference from other vehicles

Fig. 10. Mean interference as a function of the antenna angle in highway scenario.

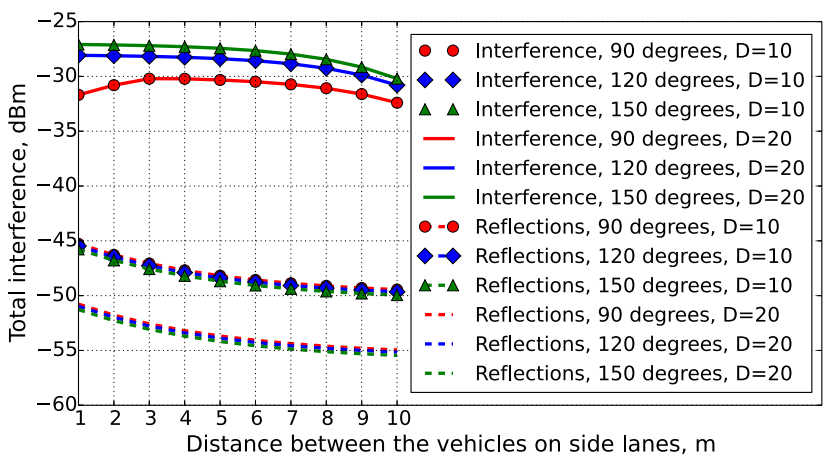

(a) Total interference

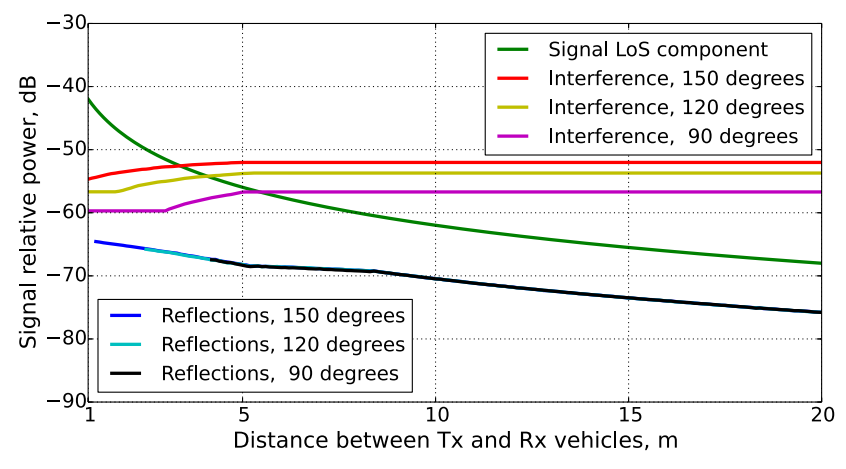

(b) Signal relative power

Fig. 11. Total interference and signal attenuation as functions of inter-vehicle distance in highway scenario.

4) Link-Level Performance Metrics: Once the mean values of URSS, $E[P]$, multipath interference, $E\left[P_{I}\right]$, and interference from other vehicles, $E[I]$, are obtained, the link level performance analysis follows the procedure described for the deterministic case. Particularly, we first obtain the mean SINR, $E[S]$, and the proceed approximating the mean special efficiency, $E[U]$, and the mean capacity $E[C]$ using the Taylor series approximation according to (25).

\section{Numerical Results}

In this section, we first characterize the interference level in both highway and urban scenarios in Section V-A and Section V-B, respectively. We also validate our models from Section IV using the ray-tracing simulations. Then, we report on the difference in link performance between the $79 \mathrm{GHz}$ and $300 \mathrm{GHz}$ systems in Section V-C.

\section{A. Interference in Highway Scenario}

Consider first the mean interference in highway scenario as a function of antenna angle illustrated in Fig. 10. The mean multipath interference is presented in Fig. 10(a), while the mean interference from the concurrent transmissions is given in Fig. 10(b). To illustrate the qualitative behavior of the interference and reduce the amount of plotted data, we analyze the highway scenario focusing exclusively on $300 \mathrm{GHz}$ band.
The $79 \mathrm{GHz}$ system shows the qualitatively similar picture. In what follows, we particularly concentrate on the worst-case interference from side lanes, appearing when $W<<D$.

Analyzing Fig. 10(a), one can notice a "step function" with two jumps in the mean multipath interference level as the antenna directivity angle increases. These jumps correspond to the appearance of to the first- and second-order reflections at the Rx. The multipath interference level is zero for the small antenna angles as no reflections are feasible at the selected distances between Tx and Rx. The first-order reflection appears at the antenna angle of $\alpha=\tan ^{-1}[(2 l-R) / D]$, which equals to $45^{\circ}$ and $26^{\circ}$ for $D=10 \mathrm{~m}$ and $D=15 \mathrm{~m}$, respectively. After this point, the multipath interference remains constant until the second-order reflection becomes feasible, which happens at $81^{\circ}$ and $60^{\circ}$ for the distances of $10 \mathrm{~m}$ and $15 \mathrm{~m}$, respectively. For the selected range of $D$ we do not see any contribution of the third- and beyond order reflections, thus, the mean multipath interference remains constant. Moreover, as one may observe, the contribution of the second-order reflections is much less compared to the first-order reflections as both the power and the probability of at least one of them appearing are significantly smaller. Based on these facts, we conclude that a good estimate of the multipath interference can be obtained already with the first-order reflections only as the relative difference between first- and first-plus-second reflections is around 3\%. We also observe that the mean 
multipath interference is larger for the smaller values of $D$, as the reflected components are less attenuated. In addition, the multipath interference is, expectedly, higher for denser traffic in the neighboring lanes, as the small distance between the side vehicles increases the chances of reflections to appear.

The mean interference from the other vehicles versus the antenna directivity angle is presented in Fig. 10(b). For small values of antenna angle, the interference is zero as all the possible interference paths are blocked by the next vehicle in the lane. However, for antenna angles starting from $45^{\circ}$ and $60^{\circ}$ for $W=2 \mathrm{~m}$ and $W=1 \mathrm{~m}$, respectively, the interference appears, since the interfering components are no more blocked by the vehicles' bodies. After this point, the interference starts growing being simultaneously affected by three contradictory trends. On one side, the greater antenna directivity angle leads to higher chances of the vehicle on the next lane to interfere with the transmission of interest, moreover, the greater the antenna directivity angle, the relatively shorter becomes the average path for the interfering component. At the same time, the growth of the average interference is partly suppressed by the fact that the greater the antenna directivity angle is the lower is the relative gain, so the smaller fraction of energy will be transmitted to a given direction. Due to this suppression, the interference starts growing slower with the values of the antenna directivity angle becoming close to $180^{\circ}$. There is also a small jump from zero to a non-zero positive value around $45^{\circ}$ and $60^{\circ}$ for $W=2 \mathrm{~m}$ and $W=1 \mathrm{~m}$, due to the fact that the process of average interference growth with the antenna directivity angle is smooth only assuming that the vehicles' bodies are transparent. In more realistic set of assumptions, as the ones incorporated in our work, the smooth growth of average interference is also affected by the "step function"shape blockage, totally cutting the interference from neighbor lanes for antenna directivity angle smaller than a certain value.

Note that the mean interference from the other vehicles is almost two orders of magnitude higher than the multipath interference level. Therefore, we conclude that in the presence of notable interference from the other vehicles, the multipath interference effect can be totally excluded from consideration in highway scenario. One may also notice a good match between the modeling and ray-based simulation results for both multipath interference and aggregated interference from the other vehicles. Thus, in the following discussion on highway scenario, we will rely on the model results only.

Having revealed the principal structure of interference in highway scenario, we now proceed illustrating its special effects. Fig. 11(a) shows the mean interference in $\mathrm{dBm}$ as a function of the distance between vehicles on side lanes, $W$. Observe that the mean interference from the other vehicles does not depend on the distance between vehicles on the central lane, $D$, for all values of $W$, while the mean multipath interference, expectedly, does. Secondly, the interference from the other vehicles has a maximum value, where the interfering transmissions have, in average, relatively high chances not to be blocked by the neighboring vehicles and, at the same time, they are initiated not very far from the $\mathrm{Rx}$ vehicle. For $90^{\circ}$ antenna the maximum is at $W=3 \mathrm{~m}$, while for both $120^{\circ}$ and $150^{\circ}$ angles it is at less than $1 \mathrm{~m}$.
The received power for LoS component, multipath interference due to reflections and interference from other vehicles as a function of the distance between $\mathrm{Tx}$ and $\mathrm{Rx}$ vehicles, $D$, is shown in Fig. 11(b). As one may observe, the signal relative power, expectedly, decreases with distance. Since the reflected components need to travel longer distances and the corresponding incident angles increase, the net effect is greater losses for LoS and the multipath interference components. This illustration also highlights the points, where the multipath interference starts to appear, that is, multipath interference is zero until $1.2 \mathrm{~m}, 2 \mathrm{~m}$, and $4.2 \mathrm{~m}$ for $150^{\circ}, 120^{\circ}$, and $90^{\circ}$, respectively. We also see that the curves change their behavior around $8 \mathrm{~m}$, where the second-order reflections become feasible. The mean interference level slowly grows with the distance for all three angles until it reaches the maximum level. This level is defined by the point, where the interfering signals are blocked by the Tx car. In this case, even though it is theoretically feasible to have an infinite number of interfering vehicles on side lanes, the aggregated contribution of vehicles located far behind the Tx vehicle is marginal, as the distance between them and the $\mathrm{Rx}$ vehicle also grows.

\section{B. Interference in Urban Scenario}

We now proceed with the numerical characterization of the interference in the urban (stochastic) scenario. Due to space constraints, we do not present the individual contributions of multipath interference and the interference from the other vehicles. In Fig. 12(a), we focus on the total interference at the receiver as a function of the antenna angle and the density of traffic. As one may notice, the total level of interference in urban scenario also grows with the antenna angle. However, as opposed to the highway scenario featuring a number of jumps, the curves are now smooth. The reason is that both the appearance of certain reflections, as well as the distances of interference paths, are now probabilistic.

The mean value of inter-car distance, $W$, characterizing the traffic density, plays an important role in the total interference picture. Depending on the traffic density, the total interference at the receiver could be up to $10 \mathrm{~dB}$ different for $79 \mathrm{GHz}$ system. For instance, it grows from $-55 \mathrm{dBm}$ to $-47 \mathrm{dBm}$ for the antenna angle of $60^{\circ}$. However, the same difference for the $300 \mathrm{GHz}$ system is just $4 \mathrm{~dB}$, as the signal at these frequencies is much more attenuated with distance. Generalizing these conclusions, we notice that the traffic density affects the behavior of the $300 \mathrm{GHz}$ system much less in the entire range of the antenna angles, while the total interference level is around 10$15 \mathrm{~dB}$ lower than for $79 \mathrm{GHz}$, as the signal at $300 \mathrm{GHz}$ is not only affected by higher path loss but is characterized by higher reflection losses, estimated in Section IV.

We now continue with Fig, 12(b), presenting the mean value of signal-to-interference ratio as a function of antenna angle. Analyzing this figure, we first observe the match between the curves for $79 \mathrm{GHz}$ and $300 \mathrm{GHz}$ setups. This observation follows the expectations as the SIR metric, by design, shows the ratio between the received signal strength and the interference. Both useful signal and interfering signals propagate in identical environments, therefore, the frequency-dependent 


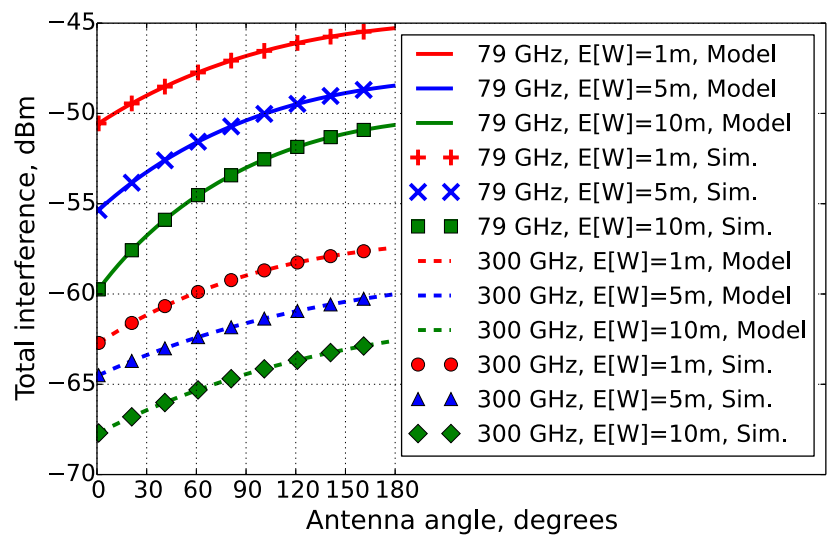

(a) Total interference

Fig. 12. Mean interference and SIR in urban scenario.

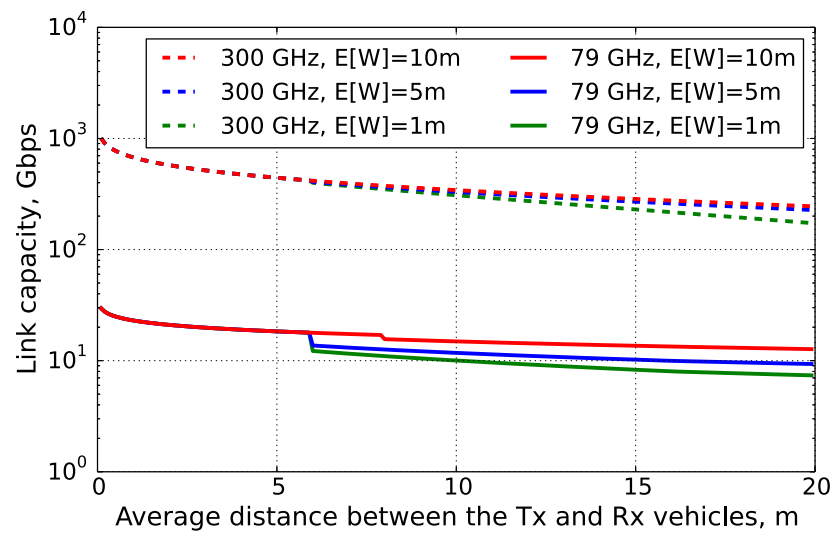

(a) Urban scenario

Fig. 13. Achievable link capacity in highway and urban scenarios.

components cancel each other out. More importantly, this figure illustrates that even in the stochastic scenario, in the presence of interfering nodes the increase of the distance between the transmitter and the receiver requires narrower beams to guarantee the same level of link quality. For instance, $10 \mathrm{~dB}$ SIR is achievable with $90^{\circ}$ angle at the distances of around $1 \mathrm{~m}$, while for $5 \mathrm{~m}$ and $10 \mathrm{~m}$ range the angles should be not greater than $60^{\circ}$ and $50^{\circ}$, respectively.

Finally, similar to the highway scenario, we observe a close match between the analytical and simulation results, confirming the accuracy of the developed model.

\section{THz vs. mmWaves for V2V Communications}

Utilizing the developed analytical models, we now proceed characterizing the interference and noise effects on the link capacity. We assume that the $79 \mathrm{GHz}$ system use $1 \mathrm{GHz}$ bandwidth, while the $300 \mathrm{GHz}$ system operates over much wider $50 \mathrm{GHz}$ spectrum. The Tx power is equal for both systems and set to $20 \mathrm{dBm}$.

We first focus on the urban scenario. Fig. 13(a) presents the mean link capacity for the considered communications system as a function of the average distance between the $\mathrm{Tx}$ and $\mathrm{Rx}$ vehicles as well as the average distance between the vehicles on the side lanes, $E[W]$. We incorporate both the effects of multipath interference and interference from other vehicles as

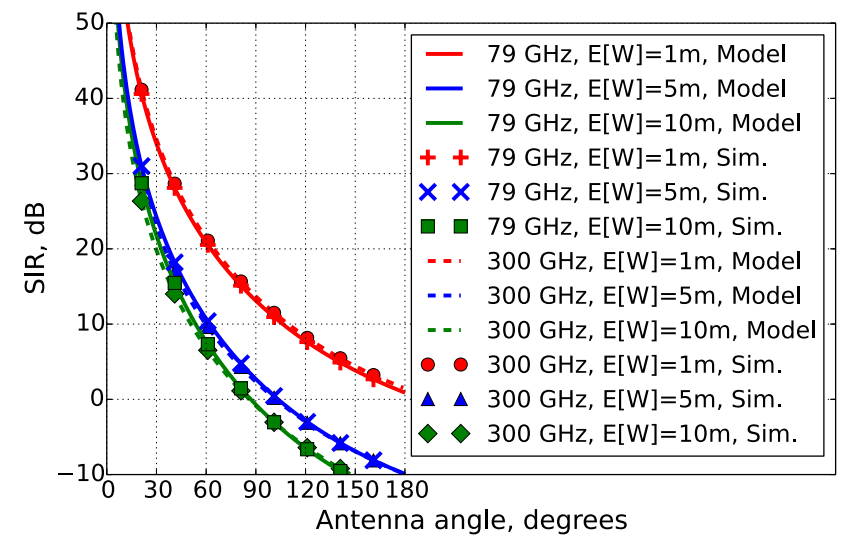

(b) Signal-to-Interference ratio

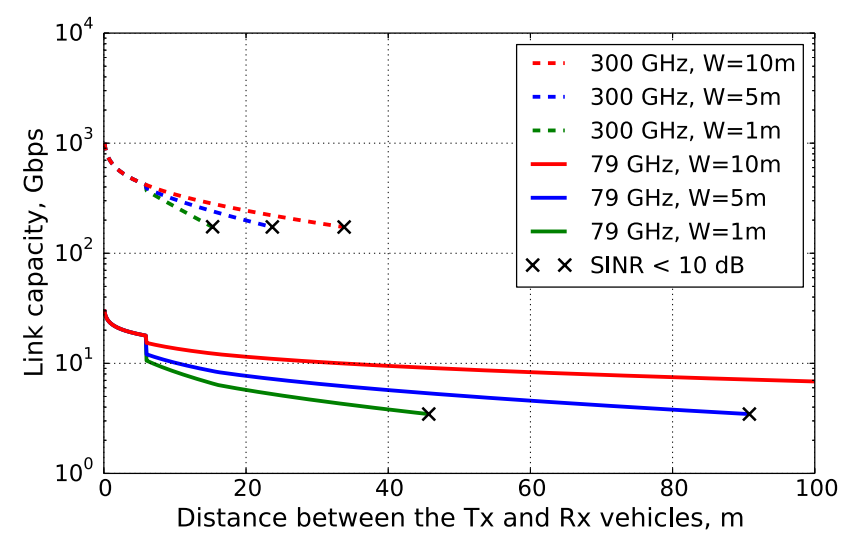

(b) Highway scenario

well as the thermal noise. Analyzing the results, we observe a clear advantage of $300 \mathrm{GHz}$ band over $79 \mathrm{GHz}$ band for the considered range of communication distances ( $0 \mathrm{~m}$ to $20 \mathrm{~m}$ ), which we believe are realistic for dense urban environments. As the values of SIR are close, as shown in Fig. 12(b), and the received signal strength for both frequency ranges is still notably greater than the thermal noise level, the $300 \mathrm{GHz}$ system clearly outperforms the competing $79 \mathrm{GHz}$ one in terms of the link capacity due to 50 times wider bandwidth.

Continuing with the highway scenario, Fig. 13(b) presents the mean link capacity for the considered communications system as a function of the distance between the $\mathrm{Tx}$ and Rx vehicles, $D$, as well as the distance between the vehicles on the side lanes, $W$. Here, we limit the capacity curves to the $\operatorname{SINR} \geq 10 \mathrm{~dB}$ regime only, as high link capacity is useless if it is difficult to utilize it due to the poor channel quality. Comparing the urban and highway scenario, we have to admit a notable advantage of the former due to the lower level of interference from other vehicles, see Fig. 12(a) versus Fig. 11(a), which results in greater link capacity, see Fig. 13. Also, on the contrary to the highway scenario, mean SIR never dropped lower than $10 \mathrm{~dB}$ threshold for the considered range of communication distances (from $0 \mathrm{~m}$ to $20 \mathrm{~m}$ ).

As one can observe from Fig. 13(b), the reliable link distance for $300 \mathrm{GHz}$ system is limited to $34 \mathrm{~m}$, while the 
same value for $79 \mathrm{GHz}$ can easily exceed $100 \mathrm{~m}$. At the same time, $300 \mathrm{GHz}$ system has greater capacity due to the wider bandwidth and lower level of interference. On the contrast, the $79 \mathrm{GHz}$ system expects notable interference after $D \approx 5 \mathrm{~m}$ as the concurrent transmissions at the side lanes are no longer blocked by the vehicles. As expected, the denser scenarios lead to worse performance for both $300 \mathrm{GHz}$ and $79 \mathrm{GHz}$ systems. The performed comparison clearly illustrates that the millimeter wave systems operating at $79 \mathrm{GHz}$ is preferred for the long-range communications (especially on the highways), while the $300 \mathrm{GHz}$ solutions are better suited for shorter-range bandwidth-greedy applications in more dense environments.

\section{CONCLUSiOnS}

In this paper, using the results of the measurements campaign and relying on the tools of stochastic geometry, the analytical models for interference caused by the vehicles on the side lanes in mmWave/ $\mathrm{THz} \mathrm{V} 2 \mathrm{~V}$ communications have been formulated for both highway and urban scenarios.

Our results indicate that interference from the side lanes in the considered deployments can be well approximated by 2D stochastic models. In addition, the interference level in the analyzed configurations greatly depends on the angle of the antenna radiation pattern. Particularly, for angles greater than $40^{\circ}$, the interference caused by the vehicles on the side lanes become a critical issue that has to be taken into account. On the contrary, for angles smaller than $20^{\circ}$ the interference in dense deployments of vehicles is typically marginal even in the absence of the interference mitigation techniques.

The presented study characterizes a notable source of interference in $\mathrm{V} 2 \mathrm{~V}$ communications and estimates its implications on the link layer performance of mmWave/ $\mathrm{THz}$ band $\mathrm{V} 2 \mathrm{~V}$ communication systems, thus, providing an important step towards the comprehensive characterization of the interference picture in $5 \mathrm{G}$ and beyond vehicular networks.

\section{REFERENCES}

[1] Volkswagen AG et al., "5G automotive vision," 5G Infrastructure Public Private Partnership (5G PPP), White paper, October 2015.

[2] METIS, "Updated scenarios, requirements and KPIs for 5G mobile and wireless system with recommendations for future investigations," Deliverable D1.5, April 2015.

[3] S. Andreev, V. Petrov, M. Dohler, and H. Yanikomeroglu, "Future of ultra-dense networks beyond $5 \mathrm{~g}$ : Harnessing heterogeneous moving cells," CoRR, vol. abs/1706.05197, 2017. [Online]. Available: http: //arxiv.org/abs/1706.05197

[4] G. Karagiannis, O. Altintas, E. Ekici, G. Heijenk, B. Jarupan, K. Lin, and T. Weil, "Vehicular networking: A survey and tutorial on requirements, architectures, challenges, standards and solutions," IEEE Communications Surveys \& Tutorials, vol. 13, no. 4, pp. 584-616, 2011.

[5] E. Lee, E. K. Lee, M. Gerla, and S. Y. Oh, "Vehicular cloud networking: architecture and design principles," IEEE Communications Magazine, vol. 52, no. 2, pp. 148-155, February 2014.

[6] P. Kumari, N. Gonzalez-Prelcic, and R. W. Heath, "Investigating the IEEE 802.11ad standard for millimeter wave automotive radar," in Proc. of IEEE 82nd Vehicular Technology Conference (IEEE VTC2015-Fall), September 2015.

[7] L. Reichardt, C. Sturm, F. Grunhaupt, and T. Zwick, "Demonstrating the use of the IEEE $802.11 \mathrm{p}$ car-to-car communication standard for automotive radar," in Proc. of European Conference on Antennas and Propagation (EuCAP), March 2012.

[8] V. Petrov, A. Samuylov, V. Begishev, D. Moltchanov, S. Andreev, K. Samouylov, and Y. Koucheryavy, "Vehicle-based relay assistance for opportunistic crowdsensing over Narrowband IoT (NB-IoT)," IEEE Internet of Things Journal, vol. PP, no. 99, pp. 1-1, 2017.
[9] C. Perfecto, J. D. Ser, and M. Bennis, "Millimeter-wave V2V communications: Distributed association and beam alignment," IEEE Journal on Selected Areas in Communications, vol. 35, no. 9, pp. 2148-2162, September 2017.

[10] V. Va, T. Shimizu, G. Bansal, and R. Heath, "Millimeter wave vehicular communications: A survey," Foundations and Trends in Networking, vol. 10, no. 1, pp. 1-113, June 2016.

[11] Y. Li, D. Jin, Z. Wang, L. Zeng, and S. Chen, "Coding or Not: Optimal mobile data offloading in opportunistic vehicular networks," IEEE Transactions on Intelligent Transportation Systems, vol. 15, no. 1, pp. 318-333, February 2014.

[12] National Highway Traffic Safety Administration, "Advance notice of proposed rulemaking (ANPRM)," USA Department of Transportation, Tech. Rep., 2014. [Online]. Available: http://www.nhtsa.gov/staticfiles/ rulemaking/pdf/V2V/V2V-ANPRM_081514.pdf

[13] S. Mumtaz, K. M. S. Huq, M. I. Ashraf, J. Rodriguez, V. Monteiro, and C. Politis, "Cognitive vehicular communication for 5G," IEEE Communications Magazine, vol. 53, no. 7, pp. 109-117, July 2015.

[14] J. Choi, V. Va, N. Gonzalez-Prelcic, R. Daniels, C. R. Bhat, and R. W. Heath, "Millimeter-wave vehicular communication to support massive automotive sensing," IEEE Communications Magazine, vol. 54, no. 12, pp. 160-167, December 2016.

[15] V. Va, T. Shimizu, G. Bansal, and R. W. Heath, "Beam design for beam switching based millimeter wave vehicle-to-infrastructure communications," in Proc. of IEEE International Conference on Communications (IEEE ICC), May 2016.

[16] H. Harada, K. Sato, and M. Fujise, "A radio-on-fiber based millimeterwave road-vehicle communication system by a code division multiplexing radio transmission scheme," IEEE Transactions on Intelligent Transportation Systems, vol. 2, no. 4, pp. 165-179, December 2001.

[17] E. Ben-Dor, T. S. Rappaport, Y. Qiao, and S. J. Lauffenburger, "Millimeter-wave $60 \mathrm{GHz}$ outdoor and vehicle AOA propagation measurements using a broadband channel sounder," in Proc. of IEEE Global Telecommunications Conference (GLOBECOM), December 2011.

[18] J. F. Mologni, A. S. Nunes, C. L. R. Siqueira, D. L. Figueiredo, J. C. Ribas, A. C. S. Junior, and M. A. R. Alves, "Challenges on the design of automotive radar systems and V2V technology," SAE International, Tech. Rep., September 2014. [Online]. Available: http://dx.doi.org/10.4271/2014-36-0356

[19] J. Hasch, E. Topak, R. Schnabel, T. Zwick, R. Weigel, and C. Waldschmidt, "Millimeter-wave technology for automotive radar sensors in the $77 \mathrm{GHz}$ frequency band," IEEE Transactions on Microwave Theory and Techniques, vol. 60, no. 3, pp. 845-860, March 2012.

[20] Y. Han, E. Ekici, H. Kremo, and O. Altintas, "Automotive radar and communications sharing of the 79-GHz band," in Proc. of the First ACM International Workshop on Smart, Autonomous, and Connected Vehicular Systems and Services, ser. CarSys' 16. New York, NY, USA: ACM, October 2016

[21] Engineering and Physical Sciences Research Council. (2014) Terahertz technology for future road vehicles. [Online]. Available: http: //gow.epsrc.ac.uk/NGBOViewGrant.aspx?GrantRef=EP/L019078/1

[22] V. Petrov, D. Moltchanov, and Y. Koucheryavy, "Applicability assessment of terahertz information showers for next-generation wireless networks," in Proc. of IEEE International Conference on Communications (ICC), May 2016.

[23] M. R. Akdeniz, Y. Liu, M. K. Samimi, S. Sun, S. Rangan, T. S. Rappaport, and E. Erkip, "Millimeter wave channel modeling and cellular capacity evaluation," IEEE Journal on Selected Areas in Communications, vol. 32, no. 6, pp. 1164-1179, June 2014.

[24] V. Petrov, A. Pyattaev, D. Moltchanov, and Y. Koucheryavy, "Terahertz band communications: Applications, research challenges, and standardization activities," in Proc. of 8th International Congress on Ultra Modern Telecommunications and Control Systems and Workshops (ICUMT), October 2016, pp. 183-190.

[25] T. S. Rappaport, S. Sun, R. Mayzus, H. Zhao, Y. Azar, K. Wang, G. N. Wong, J. K. Schulz, M. Samimi, and F. Gutierrez, "Millimeter wave mobile communications for $5 \mathrm{G}$ cellular: It will work!" IEEE Access, vol. 1, pp. 335-349, May 2013.

[26] J. G. Andrews, S. Buzzi, W. Choi, S. V. Hanly, A. Lozano, A. C. K. Soong, and J. C. Zhang, "What will 5G be?" IEEE Journal on Selected Areas in Communications, vol. 32, no. 6, pp. 1065-1082, June 2014.

[27] V. Petrov, M. Komarov, D. Moltchanov, J. M. Jornet, and Y. Koucheryavy, "Interference analysis of EHF/THF communications systems with blocking and directional antennas," in Proc. of IEEE Global Communications Conference (GLOBECOM), December 2016. 
[28] J. B. Kenney, "Dedicated short-range communications (DSRC) standards in the United States," Proceedings of the IEEE, vol. 99, no. 7, pp. 11621182, July 2011.

[29] C. Campolo, A. Vinel, A. Molinaro, and Y. Koucheryavy, "Modeling broadcasting in IEEE 802.11p/WAVE vehicular networks," IEEE Communications Letters, vol. 15, no. 2, pp. 199-201, February 2011.

[30] X. Cheng et al., "Wideband channel modeling and intercarrier interference cancellation for vehicle-to-vehicle communication systems," IEEE Journal on Selected Areas in Communications, vol. 31, no. 9, pp. 434 448, September 2013.

[31] D. W. Matolak, I. Sen, and W. Xiong, "Channel modeling for V2V communications," in Proc. of the 3rd Annual International Conference on Mobile and Ubiquitous Systems - Workshops, July 2006.

[32] C. B. Liu, B. Sadeghi, and E. W. Knightly, "Enabling vehicular visible light communication (V2LC) networks," in Proc. of ACM International Workshop on Vehicular Inter-networking, ser. VANET '11, January 2011.

[33] F. Schmidt-Eisenlohr, "Interference in vehicle-to-vehicle communication networks - Analysis, modeling, simulation and assessment," Ph.D. dissertation, Karlsruhe Institute of Technology, 2010.

[34] L. Liang, H. Peng, G. Y. Li, and X. Shen, "Vehicular communications: A physical layer perspective," IEEE Transactions on Vehicular Technology, vol. 66, no. 12, pp. 10647-10659, December 2017.

[35] R. Schneider, D. Didascalou, and W. Wiesbeck, "Impact of road surfaces on millimeter-wave propagation," IEEE Transactions on Vehicular Technology, vol. 49, no. 4, pp. 1314-1320, July 2000.

[36] EU CEDR's TG Road Safety. (2010, April) Safe distance between vehicles. [Online]. Available: http://www.cedr.eu/download/Publications/ 2010/e_Distance_between_vehicles.pdf

[37] V. Petrov, D. Solomitckii, A. Samuylov, M. A. Lema, M. Gapeyenko, D. Moltchanov, S. Andreev, V. Naumov, K. Samouylov, M. Dohler, and Y. Koucheryavy, "Dynamic Multi-Connectivity performance in ultradense urban mmWave deployments," IEEE Journal on Selected Areas in Communications, vol. 35, no. 9, pp. 2038-2055, September 2017.

[38] J. M. Jornet and I. F. Akyildiz, "Channel modeling and capacity analysis for electromagnetic wireless nanonetworks in the Terahertz Band," IEEE Transactions on Wireless Communications, vol. 10, no. 10, pp. 32113221, October 2011.

[39] P. Boronin, V. Petrov, D. Moltchanov, Y. Koucheryavy, and J. M. Jornet, "Capacity and throughput analysis of nanoscale machine communication through transparency windows in the terahertz band," Nano Communication Networks, vol. 5, no. 3, pp. 72 - 82, 2014.

[40] C. Han, A. O. Bicen, and I. F. Akyildiz, "Multi-ray channel modeling and wideband characterization for wireless communications in the terahertz band," IEEE Transactions on Wireless Communications, vol. 14, no. 5, pp. 2402-2412, 2015.

[41] V. Petrov, D. Moltchanov, and Y. Koucheryavy, "On the efficiency of spatial channel reuse in ultra-dense THz networks," in Proc. of IEEE Global Communications Conference (GLOBECOM), December 2015.

[42] J. M. Jornet and I. F. Akyldiz, "Femtosecond-long pulse-based modulation for terahertz band communication in nanonetworks," IEEE Transactions on Communications, vol. 62, no. 5, pp. 1742-1754, May 2014.

[43] Harvard-Smithson Center for Astrophysics. (2014) HITRAN: Highresolution transmission molecular absorption database. [Online]. Available: www.cfa.harvard.edu

[44] S. Singh, R. Mudumbai, and U. Madhow, "Interference analysis for highly directional $60-\mathrm{GHz}$ mesh networks: The case for rethinking medium access control," IEEE/ACM Transactions on Networking, vol. 19 , no. 5, pp. 1513-1527, October 2011.

[45] V. Petrov, M. Komarov, D. Moltchanov, J. M. Jornet, and Y. Koucheryavy, "Interference and SINR in millimeter wave and terahertz communication systems with blocking and directional antennas," IEEE Transactions on Wireless Communications, vol. 16, no. 3, pp. 17911808, March 2017.

[46] TeraView. (2016) Terahertz equipment for imaging and spectroscopy. [Online]. Available: http://www.teraview.com/products/index.html

[47] S. M. Ross, Introduction to probability models, 10th ed. Academic Press, 2009.

[48] M. Abramowitz and I. Stegun, Handbook of Mathematical Functions with Formulas, Graphs, and Mathematical Tables. Dover, 1965.

[49] F. Baccelli and B. Blaszczyszyn, Stochastic Geometry and Wireless Networks, Part I: Theory. Now Publishers, 2009.

[50] H. ElSawy, E. Hossain, and M. Haenggi, "Stochastic geometry for modeling, analysis, and design of multi-tier and cognitive cellular wireless networks: A survey," IEEE Communications Surveys Tutorials, vol. 15, no. 3, pp. 996-1019, July 2013.

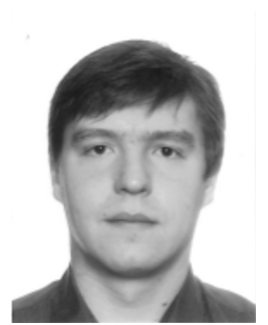

Vitaly Petrov (vitaly.petrov@tut.fi) received the M.Sc. degree in information systems security from the Saint Petersburg State University of Aerospace Instrumentation, St Petersburg, Russia, in 2011, and the M.Sc. degree in communications engineering from the Tampere University of Technology, Tampere, Finland, in 2014, where he is currently pursuing the Ph.D. degree. His work is mainly focused on enabling terahertz band communications for Beyond-5G wireless networks. He was a Visiting Scholar with the Georgia Institute of Technology, Atlanta, USA, in 2014 and a Strategic Intern with the Nokia Research Center, Helsinki, Finland, in 2012. He is the recipient of Best Student Paper Award at IEEE VTC-Fall'15 and Best Student Poster Award at IEEE WCNC'17. His current research interests are in Internet-of-Things, terahertz band communications, nanonetworks, cryptology, and network security.

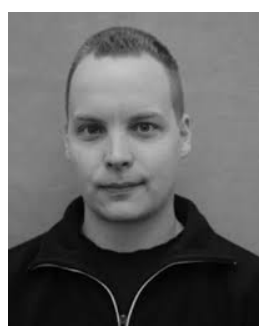

Joonas Kokkoniemi (joonas.kokkoniemi@oulu.fi) received the B.Sc. and M.Sc. degrees in electrical engineering from University of Oulu, Finland, in 2011 and 2012, respectively, where he is currently pursuing the Dr.Sc. degree with the Centre for Wireless Communications. In 2013, he was a Visiting Researcher with the Tokyo University of Agriculture and Technology, Tokyo, Japan. His main research interests are in $\mathrm{THz}$ band nanocommunications, nanonetworks, and channel modeling.

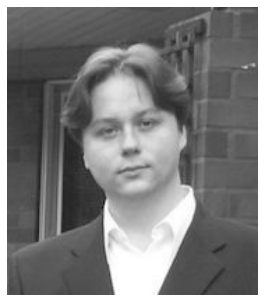

Dmitri Moltchanov (dmitri.moltchanov@tut.fi) is a Senior Research Scientist in the Laboratory of Electronics and Communications Engineering, Tampere University of Technology, Finland. He received his M.Sc. and Cand.Sc. degrees from Saint-Petersburg State University of Telecommunications, Russia, in 2000 and 2002, respectively, and Ph.D. degree from Tampere University of Technology in 2006. His research interests include performance evaluation and optimization issues of wired and wireless IP networks, Internet traffic dynamics, quality of user experience of real-time applications, and traffic localization P2P networks. Dmitri Moltchanov serves as TPC member in a number of international conferences. He authored more than 50 publications.

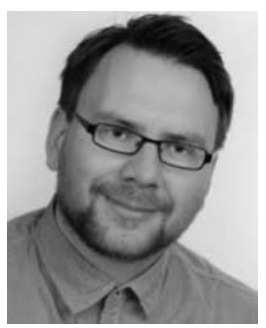

Janne Lehtomäki (janne.lehtomaki@oulu.fi) received the Ph.D. degree from the University of Oulu, Finland, in 2005. He was with Georgia Tech, Atlanta, GA, USA, as a Visiting Scholar in 2013. He is currently an Adjunct Professor with the Centre for Wireless Communications, University of Oulu. He is focusing on spectrum measurements and terahertz band wireless communication. He co-authored the paper receiving the Best Paper Award at the IEEE WCNC in 2012. He is an Editorial Board Member of Physical Communication. He was a General CoChair of the IEEE WCNC 2017 International Workshop on Smart Spectrum, a TPC Co-Chair of the IEEE WCNC 2015 and 2016 International Workshop on Smart Spectrum, and a Publicity/Publications Co-Chair of the ACM NANOCOM in 2015, 2016, and 2017. He has served as a Guest Associate Editor of the IEICE Transactions on Communications Special Section from 2014 to July 2017, and as a Managing Guest Editor of the Nano Communication Networks Special Issue in 2016. 


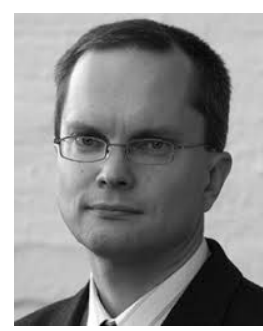

Markku Juntti (markku.juntti@oulu.fi) received the M.Sc. and Dr.Sc. degrees in electric engineering from the University of Oulu, Oulu, Finland, in 1993 and 1997, respectively.He was with the University of Oulu from 1992 to 1998. From 1994 to 1995, he was a Visiting Scholar with Rice University, Houston, TX, USA. From 1999 to 2000, he was a Senior Specialist with Nokia Networks. He has been a Professor of communications engineering with the Centre for Wireless Communications, Department of Communication Engineering, University of Oulu, since 2000. From 2014 to 2017, he also serves as the Dean of University of Oulu Graduate School. He is also an Adjunct Professor with the Department of Electrical and Computer Engineering, Rice University. His research interests include signal processing for wireless networks and communication and information theory. He has authored or co-authored 350 papers published in international journals and conference records and books WCDMA for UMTS and Signal Processing Handbook.

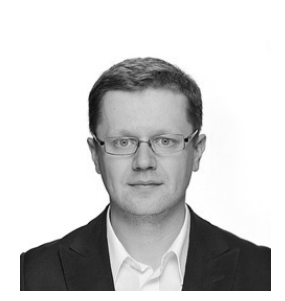

Yevgeni Koucheryavy (evgeni.kucheryavy@tut.fi) received the Ph.D. degree from the Tampere University of Technology, in 2004. He is a Professor and Laboratory Director with the Laboratory of Electronics and Communications Engineering, Tampere University of Technology, Finland. He is the author of numerous publications in the field of advanced wired and wireless networking and communications. His current research interests include various aspects in heterogeneous wireless communication networks and systems, the Internet of Things and its standardization, and nanocommunications. He is an Associate Technical Editor of the IEEE Communications Magazine and Editor of the IEEE Communications Surveys and Tutorials. 